BULLETIN (New Series) OF THE

AMERICAN MATHEMATICAL SOCIETY

Volume 43, Number 1, Pages 43-73

S 0273-0979(05)01085-2

Article electronically published on November 22, 2005

\title{
NEW METHODS IN CELESTIAL MECHANICS AND MISSION DESIGN
}

\author{
JERROLD E. MARSDEN AND SHANE D. ROSS
}

To Henri Poincaré on the 150th anniversary of his birth.

Abstract. The title of this paper is inspired by the work of Poincaré [1890, 1892], who introduced many key dynamical systems methods during his research on celestial mechanics and especially the three-body problem. Since then, many researchers have contributed to his legacy by developing and applying these methods to problems in celestial mechanics and, more recently, with the design of space missions.

This paper will give a survey of some of these exciting ideas, and we would especially like to acknowledge the work of Michael Dellnitz, Frederic Gabern, Katalin Grubits, Oliver Junge, Wang-Sang Koon, François Lekien, Martin Lo, Sina Ober-Blöbaum, Kathrin Padberg, Robert Preis, and Bianca Thiere.

One of the purposes of the AMS Current Events session is to discuss work of others. Even though we were involved in the research reported on here, this short paper is intended to survey many ideas due to our collaborators and others.

This survey is by no means complete, and we apologize for not having time or space to do justice to many important and fundamental works. In fact, the results reported on here rely on and were inspired by important preceding work of many others in celestial mechanics, mission design and in dynamical systems. We mention just a few whose work had a positive influence on what is reported here: Brian Barden, Ed Belbruno, Robert Farquhar, Gerard Gómez, George Haller, Charles Jaffé, Kathleen Howell, Linda Petzold, Josep Masdemont, Vered Rom-Kedar, Radu Serban, Carles Simó, Turgay Uzer, Steve Wiggins, and Roby Wilson. In an upcoming monograph (see Koon, Lo, Marsden, and Ross [2005]), the dynamical systems and computational approach and its application to mission design are discussed in detail.

One of the key ideas is that the competing gravitational pull between celestial bodies creates a vast array of passageways that wind around the Sun, planets and moons. The boundaries of these passageways are realized geometrically as invariant manifolds attached to equilibrium points and periodic orbits in interlinked three-body problems. In particular, tube-like structures form an interplanetary transport network which will facilitate the exploration of Mercury, the Moon, the asteroids, and the outer solar system, including a mission to assess the possibility of life on Jupiter's icy moons. The use of these methods in problems in molecular dynamics of interest in chemistry is also briefly discussed.

Received by the editors May 3, 2005, and, in revised form, July 19, 2005.

2000 Mathematics Subject Classification. Primary 70F07, 70F15; Secondary 37J45, 70H33.

Key words and phrases. Three-body problem, mission design, transport, celestial mechanics.

The first author's research was supported in part by a Max Planck Research Award and NSFITR Grant ACI-0204932.

The second author's research was supported by an NSF Postdoctoral Fellowship, DMS 0402842.

This article is based on a lecture presented January 7, 2005, at the AMS Special Session on Current Events, Joint Mathematics Meetings, Atlanta, GA.

(C)2005 American Mathematical Society Reverts to public domain 28 years from publication 


\section{Contents}

1. Astrodynamics and dynamical astronomy

2. The patched three-body approximation

3. Transport in the solar system and beyond

Acknowledgements

About the authors

References

\section{AstrodynAmiCs AND DYNAMICAL ASTRONOMY}

Astrodynamics and dynamical astronomy apply the principles of mechanics, including the law of universal gravitation, to the determination of the motion of objects in space. In these subjects, orbits of astronomical bodies such as stars, planets, and asteroids are calculated, as are spacecraft trajectories, from launch through atmospheric re-entry, including all the needed orbital maneuvers.

While there are no sharp boundaries, astrodynamics has come to denote primarily the design and control of spacecraft trajectories, while dynamical astronomy is concerned with the motion of other bodies in the solar system (origin of the Moon, Kuiper belt objects, etc.) and the motion of stars in the galaxy. From the dynamical systems perspective that we have adopted, it has proven quite useful to mix these subjects rather than to isolate them. There is one obvious commonality: the model used for studying either a spacecraft or, say, the motion of an asteroid is the restricted $N+1$ body problem, where $N$ celestial bodies move under the influence of one another and the spacecraft or asteroid moves in the field of these bodies but has a mass too small to influence their motion.

The Ephemeris and Its Approximations. In the case of motion within the solar system, the motion of the $N$ bodies (planets, moons, etc.) can be measured and predicted to great accuracy, producing an ephemeris. An ephemeris is simply a listing of positions and velocities of celestial bodies as a function of time with respect to some coordinate system. An ephemeris can be considered as the solution of the $N$-body gravitational problem and forms the gravitational field which determines a spacecraft or asteroid's motion.

While the final trajectory design phase of a space mission or the long-term trajectory of an asteroid will involve a solution considering the most accurate ephemeris, insight can be achieved by considering simpler, approximate ephemerides (the plural of ephemeris). An example of such an ephemeris is a simplified solution of the $N$-body problem, where $N$ is small, for example, the motion of the Earth and Moon under their mutual gravitation, a two-body solution. The simplest two-body solution of massive bodies which gives rise to interesting motion for a spacecraft is the circular motion of two bodies around their common center of mass. The problem of such a spacecraft's motion is then known as the circular restricted three-body problem, or the CR3BP.

Introduction to the Trajectory Design Problem. The set of possible spacecraft trajectories in the three-body problem can be used as building blocks for the design of spacecraft trajectories in the presence of an arbitrary number of bodies. 


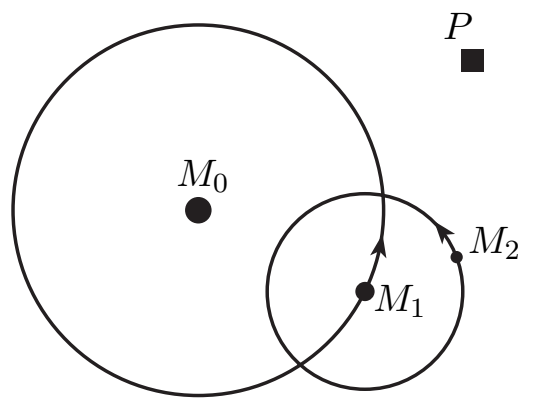

Figure 1.1. A spacecraft $P$ in the gravitational field of $N$ massive bodies which move in prescribed orbits.

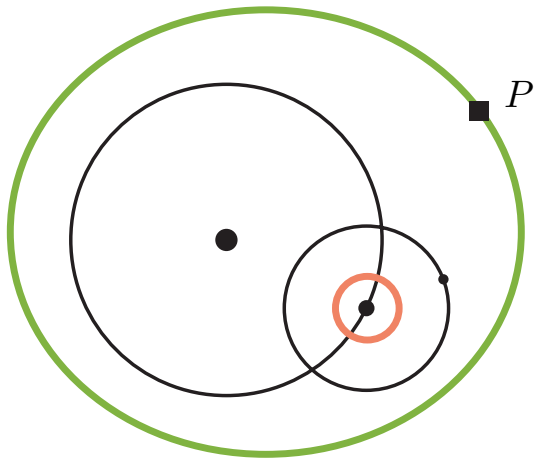

(a)

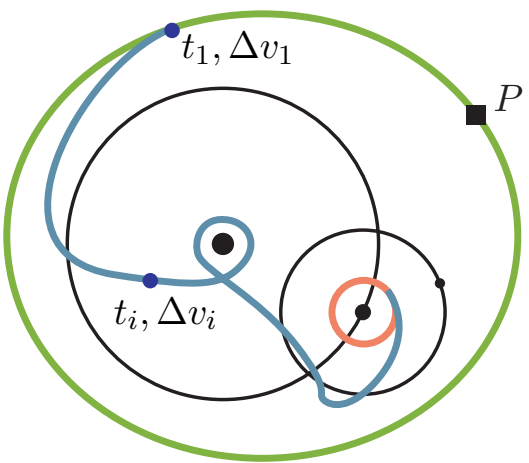

(b)

Figure 1.2. (a) The goal is to find a transfer trajectory which takes the spacecraft from an initial orbit to a final orbit using controls. (b) Assuming impulsive controls, i.e., several instantaneous changes in the spacecraft's velocity, with norm $\Delta v_{i}$ at time $t_{i}$, we can effect such a transfer.

Consider the situation shown in Figure 1.1, where we have a spacecraft, approximated as a particle, $P$, that is moving in the gravitational field of $N$ massive bodies. We assume $P$ has a small enough mass that it does not influence the motion of the $N$ massive bodies, which move in prescribed orbits under their mutual gravitational attraction. In the solar system, one can think of a moon, $M_{2}$, in orbit around a planet, $M_{1}$, which is in orbit around the Sun, $M_{0}$.

One of the goals of trajectory design is to find a transfer trajectory, such as the one shown in Figure 1.2(a), which takes the spacecraft from a prescribed initial orbit to a prescribed final orbit using controls. The initial orbit may be an orbit around the Earth and the final orbit an orbit around one of the moons of Jupiter, for instance. To effect such a transfer, one can use high thrust or low thrust propulsion systems. In the low thrust case, we have a small continuous control which can operate at all times. In the high thrust case, one typically assumes that the control is discretized into several instantaneous changes in the spacecraft's velocity. These instantaneous changes have a magnitude at time $t_{i}$ that is traditionally denoted $\Delta v_{i}$. 
Under a high thrust assumption, the $\Delta v$ 's are proportional to the fuel consumption:

$$
\Delta v=-v_{e} \frac{\Delta m}{m}
$$

where $m$ is the mass of the rocket and $\Delta m$ is the mass of propellant ejected at an exhaust velocity $v_{e}$ (see, for example, Roy [1988]). As spacecraft are limited in the amount of fuel that they can carry on-board for their journey, we often want to consider an optimal control problem: minimize the fuel consumed (equivalently, energy). In other words, we want to find the maneuver times $t_{i}$ and sizes $\Delta v_{i}$ to minimize

$$
\sum_{i} \Delta v_{i}
$$

the total change in velocity, or " $\Delta V$ " as it is called.

It is typical in the space mission design community to use the magnitude of the required $\Delta V$ as a measure of the spacecraft fuel performance. The propellant mass is a much less stable quantity as a measure of spacecraft performance, since it is dependent on the spacecraft mass and various other parameters which change frequently as the spacecraft is being built. The $\Delta V$ measure comes from astrodynamics considerations only and is independent of the mass and type of spacecraft. Thus, for a given mission objective, one generally wants to minimize $\Delta V$.

We will discuss some other optimization problems and approaches to solve them at the end of the following section.

\section{THE PATCHED THREE-BODY APPROXIMATION}

To get a spacecraft from, say, Earth to other parts of the solar system, it is necessary to find solutions for the motion of the spacecraft under the influence of $N$ bodies, a notoriously difficult problem. Furthermore, one needs to find solutions with a desired behavior, e.g., flying by the giant outer planets as Voyagers 1 and 2 did, while satisfying engineering constraints, e.g., low fuel consumption, short time of flight, low radiation dose, good observational geometry, etc.

Patched-Conic Approach and the Voyager Trajectory. For many purposes it is satisfactory to simplify the general trajectory problem by considering the gravitational force between the spacecraft and only one other body at a time. Even for the case of interplanetary transfer, this simplification will suffice for many calculations. That is, one may consider escape from or capture by a planet to be an interaction between the spacecraft and that particular planet alone, whereas the transfer process is considered an interaction between the spacecraft and the Sun alone. NASA's spectacular multiple flyby missions such as Voyager and Galileo are based on this Keplerian decomposition of the solar system, known as the patched-conic approximation (or patched two-body approximation), discussed in Bate, Mueller, and White [1971].

The strategy of the designers of the Voyager missions was to initially approximate the full $N$-body solution of the spacecraft's motion as a linkage of several two-body solutions, namely, the well-known conic solutions discovered by Kepler. The spacecraft's trajectory as it coasted between two planets was considered as a heliocentric hyperbolic trajectory. The heliocentric trajectory was cleverly chosen to come close to the destination planet in order to fly by it. When the spacecraft 
came within the "sphere of influence" 1 of a planet, it was considered as a hyperbolic conic section trajectory centered on the planet. This patched-conic solution could be used as an initial guess for a numerical procedure which produced a fully integrated $N$-body solution.

High vs. Low Relative Velocities. For missions such as Voyager and Galileo, the speed of the spacecraft relative to the bodies is high, and therefore the time during which the acceleration on the spacecraft due to two bodies is comparable is very short and results in a minor perturbation away from a conic solution. But when one needs to deal with the unpropelled, or ballistic, capture regime of motion, ${ }^{2}$ where the relative speed is low, a three-body decomposition of the solar system is necessary.

Some Missions Cannot Be Approximated by the Patched-Conic Approach. For Voyager and Galileo, the patched-conic approach worked very well. But as space missions have become more demanding, other approaches have become necessary. For example, the Genesis, $L_{1}$ Gateway, and multi-moon orbiter trajectories discussed below resemble solutions of the restricted three- and fourbody problems much more than two-body problems. In fact, methods based on a patched-conic approximation would have a very difficult time finding these complicated trajectories, as they are fundamentally non-Keplerian, restricted $N$-body solutions.

Taking Better Advantage of $N$-Body Dynamics. It is possible to satisfy mission constraints using spacecraft solutions which do not take advantage of the $N$-body dynamics of a system. But this may require using more fuel than is necessary. ${ }^{3}$ Worse yet, because of the fuel restrictions on interplanetary spacecraft, some missions may not be possible if only a patched-conic approach is used. An interesting example in this category, which also served as motivation for much of our group's work, is the "rescue" of a malfunctioned Japanese space mission to the Moon by Belbruno and Miller of JPL in June, 1990. The mission originally had two spacecraft, MUSES-A and MUSES-B; B was to go into orbit around the Moon, with A remaining in Earth orbit as a communications relay. But B failed, and A did not have sufficient fuel to make the journey. However, by utilizing a trajectory concept originally discovered by Belbruno in 1986, which is more energy-efficient than the one planned for B, MUSES-A (renamed Hiten) left Earth orbit in April, 1991, and reached the Moon that October. As a result, Japan became the third nation to send a spacecraft to the Moon. After a series of scientific experiments, Hiten was purposely crashed into the Moon in April, 1993. See Belbruno [2004] for additional details of this fascinating story.

An ESA (European Space Agency) mission currently under way, SMART-1, which is a mission from the Earth to the Moon, slated to last until August, 2006, uses some of the same ideas as was used in the Hiten mission. ${ }^{4}$ Its purpose is

\footnotetext{
${ }^{1}$ The sphere of influence of a planet is the radius at which the acceleration on a spacecraft due to the planet and the Sun are approximately equal (Roy [1988]).

${ }^{2}$ Ballistic capture means that no propulsion is necessary (i.e., no $\Delta V$ ) to achieve a capture orbit at the destination body. In general, this "capture" is temporary.

${ }^{3}$ For example, Dunn [1962] proposed to use a satellite for lunar farside communications by placing it in a position where it would require approximately $1500 \mathrm{~m} / \mathrm{s}$ per year for stationkeeping. A few years later, Farquhar [1966] proposed a trajectory for the same mission which used only $100 \mathrm{~m} / \mathrm{s}$ per year by taking advantage of three-body dynamics.

${ }^{4}$ See http://sci.esa.int/science-e/www/area/index.cfm?fareaid=10.
} 
the "Testing and proving of an ion drive and miniaturised instruments, along with investigations of lunar geochemistry and a search for ice at the south lunar pole."

A Hierarchy of Models. We want to make use of the natural dynamics in the solar system as much as possible; that is, we wish to take advantage of the phase space geometry, integrals of motion, and lanes of fast unpropelled travel. We envision generating a trajectory via a hierarchy of models. One starts with simple models which capture essential features of natural dynamics. One then uses simple model solutions as initial guess solutions in more realistic models. The approach described above does this conceptually, using the patched-conic approximation to generate the first guess solution. But there are regimes of motion where conics are simply not a good approximation to the motion of the spacecraft. There is much to be gained by starting with not two-body solutions, but three-body solutions to the spacecraft's motion.

The Patched Three-Body Approximation. Motivated by the Belbruno and Miller work, we consider a restricted four-body problem wherein a spacecraft moves under the influence of three massive bodies whose motion is prescribed, as shown schematically in Figure 1.1. For Belbruno and Miller, these four bodies were the Sun, the Earth, the Moon and the spacecraft.

To begin with, we restrict the motion of all the bodies to a common plane, so the phase space is only four-dimensional. As in the patched-conic approach, the patched three-body approach uses solutions obtained from two three-body problems as an initial guess for a numerical procedure which converges to a full four-body solution.

As an example of such a problem where there is no control, consider the fourbody problem where two adjacent giant planets compete for control of the same comet (e.g., Sun-Jupiter-comet and Sun-Saturn-comet). When close to one of the planets, the comet's motion is dominated by the corresponding planet's three-body dynamics. Between the two planets, the comet's motion is mostly heliocentric and Keplerian but is precariously poised between two competing three-body dynamics, leading to complicated transfer dynamics between the two adjacent planets.

When we consider a spacecraft with control instead of a comet, we can intelligently exploit the transfer dynamics to construct low energy trajectories with prescribed behaviors, such as transfers between adjacent moons in the Jovian and Saturnian systems (Lo and Ross [1998]). For example, by approximating a spacecraft's motion in the $N+1$ body gravitational field of Jupiter and $N$ of its planetsized moons by several segments of purely three-body motion - involving Jupiter, the $i$ th moon, and the spacecraft - we can design a trajectory for the spacecraft which follows a prescribed itinerary in visiting the $N$ moons. In an earlier study of a transfer from Ganymede to Europa, we found our fuel consumption for impulsive burns, as measured by the total norm of velocity displacements, $\Delta V$, to be less than half the Hohmann transfer value (Koon, Lo, Marsden, and Ross [1999]). We found this to be the case for the following example of a multi-moon orbiter tour that is shown schematically in Figure 2.1: starting beyond Ganymede's orbit, the spacecraft is ballistically captured by Ganymede, orbits it once, escapes in the direction of Europa, and ends in a ballistic capture at Europa.

One advantage of this multi-moon orbiter approach as compared with the Voyager-type flybys is the "leap-frogging" strategy. In this new approach to mission design, the spacecraft can orbit a moon for a desired number of circuits, escape 


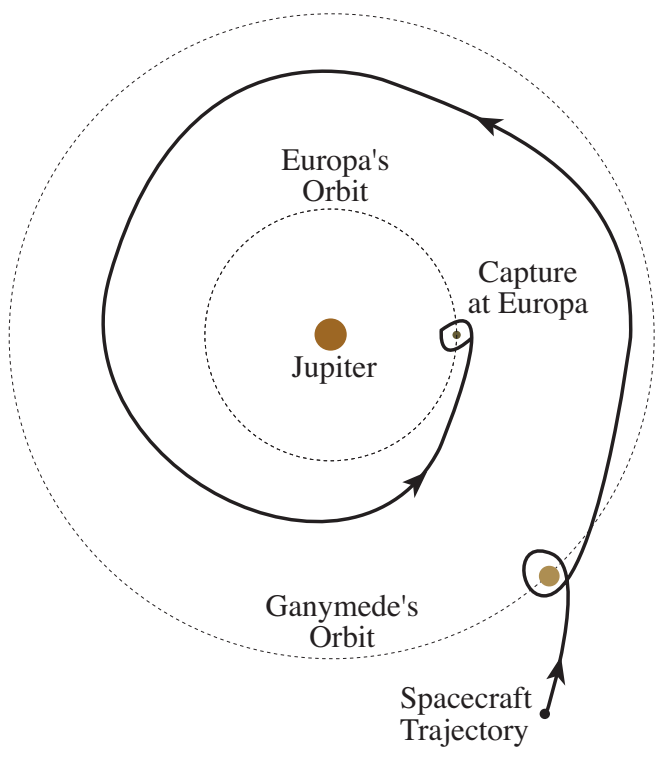

FiguRE 2.1. Leap-frogging mission concept: a multi-moon orbiter tour of Jupiter's moons Ganymede and Europa.

the moon, and then perform a transfer $\Delta V$ to become ballistically captured by a nearby moon for some number of orbits about that moon, etc. Instead of brief flybys lasting only seconds, a scientific spacecraft can orbit several different moons for any desired duration. Furthermore, the total $\Delta V$ necessary is much less than that necessary using purely two-body motion segments. One can also systematically construct low energy transfers from the Earth to the Moon using the Sun's perturbation, and from lunar libration point orbits to Earth libration point orbits.

Three-Body Dynamics. To patch three-body solutions (the spacecraft's motion in the presence of two bodies), one needs a good handle on what those solutions are. Studying the CR3BP solutions in detail is an interesting topic in its own right. This is a topic that goes back to the basic work of Poincaré in the late 1800s and provided the context in which he developed modern dynamical systems theory and the notion of chaos.

In the CR3BP, we have two primaries that move in circles; the smaller third body moves in the gravitational field of the primaries (without affecting them). We typically view the motion in a rotating frame so that the primaries appear stationary. It is important to consider both the planar and the spatial problems, but we shall focus on the planar problem for the moment.

One may derive the equations of motion using a little elementary mechanics as follows. Let the masses of the two primaries be denoted $m_{1}$ and $m_{2}$ and set $\mu=m_{2} /\left(m_{1}+m_{2}\right)$. We can normalize the distance between the primaries to be unity, and then in the rotating frame, normalized to rotate with unit angular velocity, the two bodies may be located on the $x$-axis at the points $(-\mu, 0)$ and $(1-\mu, 0)$. Let the position of the third body be denoted $(x, y)$ in the rotating frame. The kinetic energy of this third body (whose mass we take to be unity) with respect to an inertial frame but written in a frame rotating with unit angular 
velocity is the usual $\frac{1}{2} m v^{2}$ expression:

$$
K(x, y, \dot{x}, \dot{y})=\frac{1}{2}\left[(\dot{x}-y)^{2}+(\dot{y}+x)^{2}\right] .
$$

Let $r_{1}$ be the distance from the third body to the first primary and let $r_{2}$ be its distance to the second primary. Thus,

$$
\begin{aligned}
& r_{1}=\sqrt{(x+\mu)^{2}+y^{2}}, \\
& r_{2}=\sqrt{\left.(x-1+\mu)^{2}+y^{2}\right)} .
\end{aligned}
$$

Then the gravitational potential energy of the third body is, again in normalized units,

$$
V(x, y)=-\frac{1-\mu}{r_{1}}-\frac{\mu}{r_{2}}
$$

The Lagrangian of the third body is its kinetic minus potential energies, namely

$$
L(x, y, \dot{x}, \dot{y})=K(x, y, \dot{x}, \dot{y})-V(x, y) .
$$

Now one gets the equations of motion simply by writing down the corresponding Euler-Lagrange equations:

$$
\ddot{x}-2 \dot{y}=-\frac{\partial \bar{V}}{\partial x}, \quad \ddot{y}+2 \dot{x}=-\frac{\partial \bar{V}}{\partial y}
$$

where the effective potential is

$$
\bar{V}=V-\frac{x^{2}+y^{2}}{2}
$$

Being Euler-Lagrange equations, there is a conserved energy that one computes via the Legendre transformation to be

$$
E=\frac{1}{2}\left(\dot{x}^{2}+\dot{y}^{2}\right)+\bar{V}(x, y) .
$$

Equilibria. These occur when the the third body moves in a circular orbit with the same frequency as the primaries, so that it is stationary in the rotating frame. We find these points by finding the equilibrium points, in the standard sense of ode's, of the equations (2.1). It is clear that this task is equivalent to finding the critical points of the effective potential, an analysis that is found in every book on celestial mechanics. The result is that there are five such points. There are three collinear points on the $x$-axis that were discovered by Euler around 1750 and are denoted $L_{1}, L_{2}, L_{3}$, and there are two equilateral points discovered by Lagrange around 1760 and are denoted $L_{4}, L_{5}$. They are indicated in Figure 2.2.

Equations (2.1) may be interpreted as those of a particle moving in an effective potential plus a magnetic field. Its graph is shown in Figure 2.3. This figure also shows the region one gets by imposing conservation of energy and the simple inequality stating that the kinetic energy is positive. Thus, at a given energy level $E$, the third body can only move in the region given by the inequality $E-\bar{V} \geq 0$; this is called the Hill's region and is obtained by intersecting the graph of the effective potential with a horizontal plane. An example is shown as the white region in the right-hand side of Figure 2.3 for the Sun-Jupiter-third body system. In this figure, one can see three realms, namely the Sun realm, the Jupiter realm and the exterior realm that are connected by the neck regions, the left-hand neck containing $L_{1}$ and the right-hand neck containing $L_{2}$. For other values of the energy, one or more of 


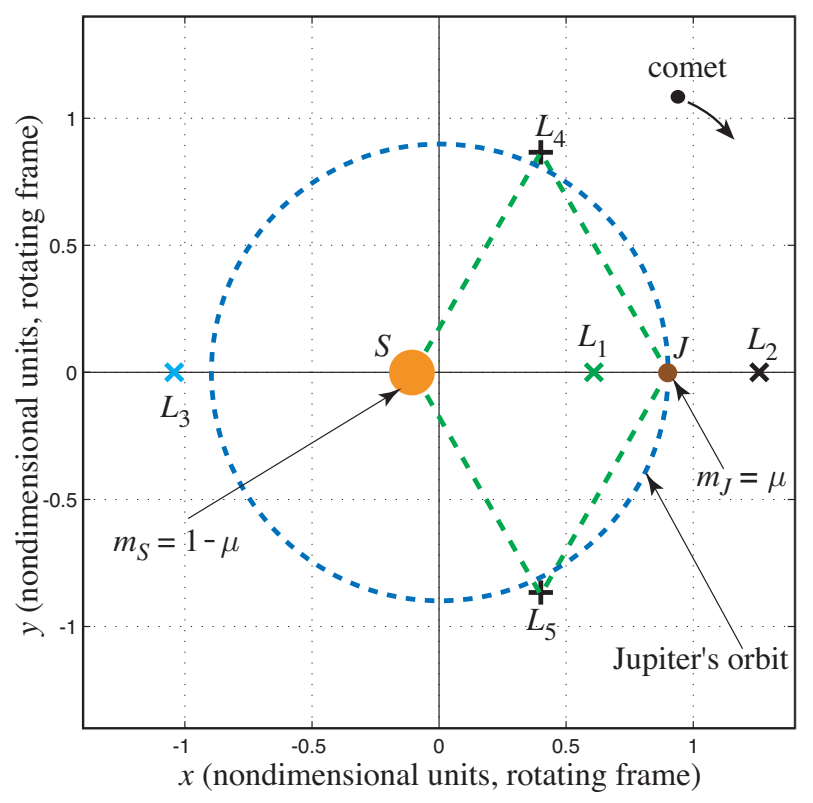

FiguRE 2.2. Equilibrium points for the three-body problem.

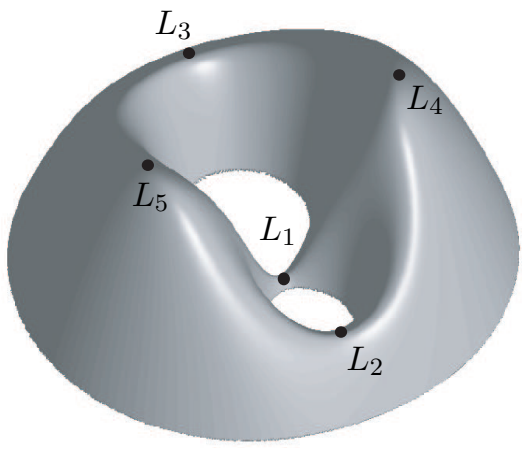

Effective Potential

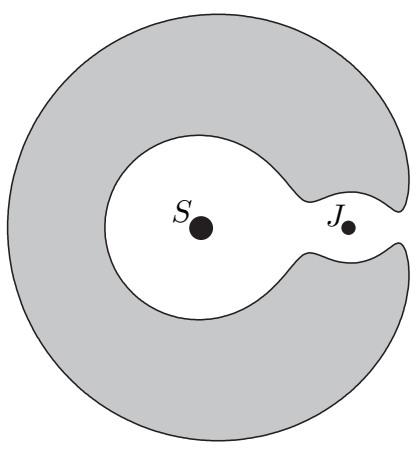

Level set shows the Hill's region

Figure 2.3. The graph of the effective potential in the three-body problem. Its critical points are the equilibria.

these realms may be prohibited due to conservation of energy; that is, the necks may close off.

Of special interest are the two points $L_{1}$ and $L_{2}$ closest to the secondary body, which a linearized analysis shows are center-saddle points. The famous Liapunov theorem says that there is a family of periodic orbits surrounding each of these points; one can think of this as meaning that one can "go into orbit about these points". These planar periodic orbits are called Liapunov orbits, while their counterparts in the $3 \mathrm{D}$ problem are called halo and Lissajous orbits (which, by the way, involves an interesting bifurcation analysis). 
Tubes. In the three-body problem, a key role is played by the invariant manifolds of these periodic orbits, which we call the Conley-McGehee tubes. Also key is a network of homoclinic and heteroclinic orbits connecting these periodic orbits, also discovered in a preliminary way in work of Conley and McGehee, which was extended and thoroughly investigated in Koon, Lo, Marsden, and Ross [2000]. Some of the reasons that these tubes are important can be seen in the context of specific space missions described below.

In fact, the invariant manifold structures of $L_{1}$ and $L_{2}$ provide the framework for understanding and categorizing the motions of spacecraft as well as, for example, comets that undergo resonance hopping. Moreover, the stable and unstable invariant manifold tubes associated to periodic orbits around $L_{1}$ and $L_{2}$ are the phase space conduits transporting material between different realms in a single three-body system as well as between primary bodies for separate three-body systems. These tubes can be used to construct new spacecraft trajectories, as we will indicate below. It is remarkable that the connecting orbits as well as the associated Conley-McGehee tubes are critical for understanding transport in the solar system as well as in molecular systems. It is quite interesting that some of the same techniques used in the celestial context can also be used in the molecular context, and conversely, techniques from chemistry can be used in celestial problems, as was done in Jaffé et al. [2002].

Figure 2.4 shows some tubes (projected from phase space to configuration space) associated with periodic orbits about $L_{1}, L_{2}$ for the Earth-Moon system. As this figure indicates, it is the tubes that control the capture and escape properties as well as transit and non-transit orbits.

Tubes in Molecular Systems. In the molecular and atomic contexts, tubes control, for instance, the scattering of electrons off Rydberg atoms, as in Figure 2.5. The Conley-McGehee tubes in this case are associated with the three degree of

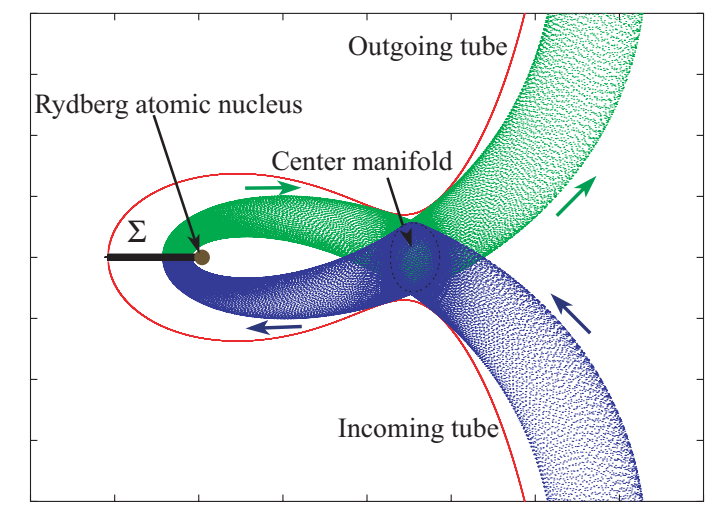

Figure 2.5. Tubes leading an electron toward and away from an ionized Rydberg atom in the presence of crossed electric and magnetic fields (seen in a frame rotating with the cyclotron frequency). Planar projections of the tubes are shown up to their first intersection with a Poincaré section, $\Sigma$. Figure taken from Gabern et al. [2005]. 


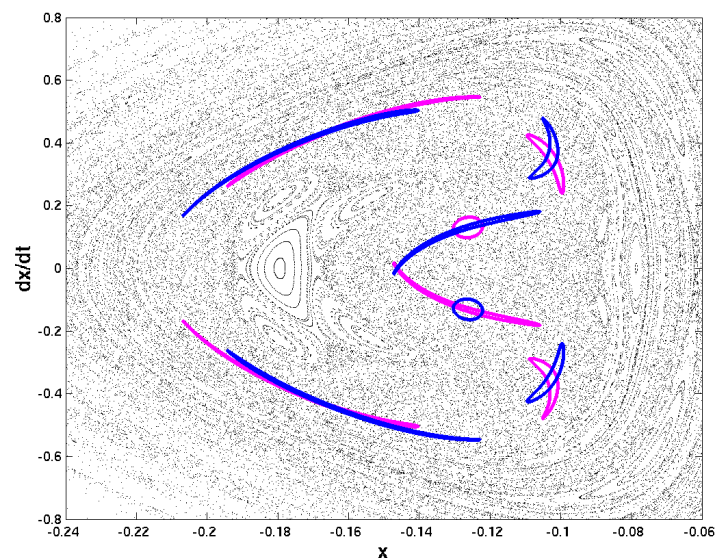

Figure 2.6. Tubes can intersect the chaotic sea multiple times, as shown in this figure, taken from Dellnitz et al. [2005], published by Turpion Ltd.; http: //www . turpion.org/php/paper . phtml?journal_id=rd\&paper_id=310. Reprinted with permission.

freedom problem counterpart of the Liapunov orbit, the set of bound orbits known which are a part of the center manifold of the equilibrium point.

Another view of tubes in the Rydberg atom problem is shown in Figure 2.6. One of the interesting things about this example is that it is one for which the classical Transition State Theory developed in chemistry needs some modifications. The basic reason is because of the complex geometry of how the tubes interact with the chaotic sea, as shown in this figure.

Building on work of Jaffé, Uzer, Koon, Lo, Marsden, Ross, Wiggins and others, the papers Gabern et al. [2005] and Dellnitz et al. [2005] develop the mathematical and computational foundations of reaction rate theory that overcome some of the classical difficulties mentioned in the preceding paragraph. There are several things that are interesting about this; first of all, it is done in a way that is synergistic with the methods that are useful in dynamical astronomy. Of course it is the underlying mathematics that provides the bridge. Another interesting thing is that these computations can be done effectively for the full three-dimensional system; the figures above are a little misleading as they seem to rely on two-dimensional Poincaré sections, which are not available for the three degree of freedom system. Nonetheless, the computations are still feasible (for the first time, actually). Current research is directed to extending these methods to more complex systems and, eventually, to conformations of biomolecules. For additional information on the celestial-molecular connection, see Porter and Cvitanović [2005].

Some Specific Missions. For the complex space missions planned for the near future, greater demands are placed on the trajectory design. In many instances, standard trajectories and classical methods such as the patched two-body approximation are inadequate to support the new mission concepts. Without appropriate and economical trajectories, these missions cannot be achieved. For nearly half a 
century, space mission planners have depended on trajectory concepts and tools developed in the 1950s and 1960s, based largely on a two-body decomposition of the solar system, the patched conics approach. While that approach remains very valuable for some missions, new trajectory paradigms must be developed to meet today's challenges.

A detailed understanding of the three-body problem, and in particular the dynamics associated with libration points, is absolutely necessary to continue the exploration and development of space.

Figure 2.7 shows, in "metro map format" some connections between hubs in Earth's neighborhood and beyond. NASA desires to develop a robust and flexible capability to visit several potential destinations. As shown in the figure, NASA has recognized that libration points $L_{1}$ and $L_{2}$ in the Sun-Earth and Earth-Moon system are important hubs and/or destinations. The fortuitous arrangement of low energy passageways in near-Earth space implies that lunar $L_{1}$ and $L_{2}$ orbits are connected to orbits around Earth's $L_{1}$ or $L_{2}$ via low energy pathways. ${ }^{5}$ Therefore, a Lunar Gateway Station at the lunar $L_{1}$ would be a natural transportation hub to get humanity beyond low-Earth orbit, a stepping stone to the Moon, Earth's neighborhood, Mars, the asteroids, and beyond. We will discuss the Lunar $L_{1}$ Gateway Station further below.

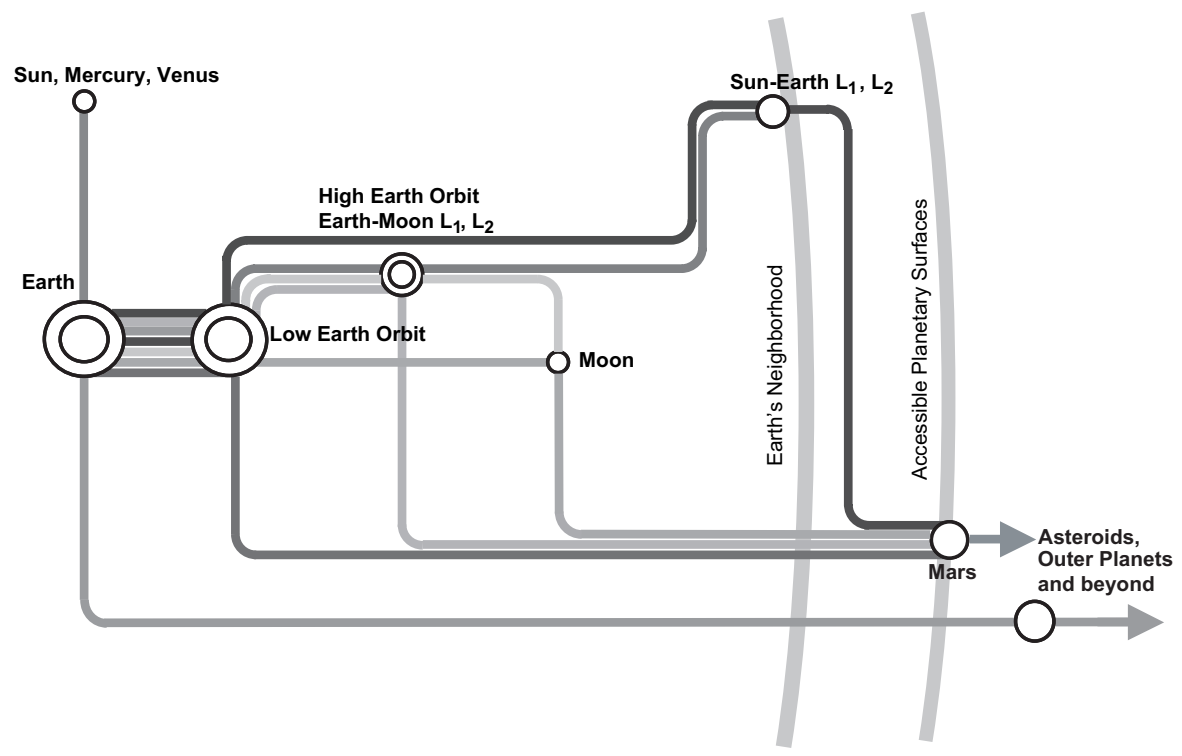

Figure 2.7. A metro map representation showing hubs connected by low energy passageways in the near-Earth neighborhood and beyond. Source: Gary L. Martin, NASA Space Architect Office.

Because of its unobstructed view of the Sun, the Sun-Earth $L_{1}$ is a good place to put instruments for doing solar science. NASA's Genesis Discovery Mission has been there, the first space mission designed completely using invariant manifolds

\footnotetext{
${ }^{5}$ We will sometimes refer to the Sun-Earth $L_{1}$ and $L_{2}$ as the Earth's $L_{1}$ and $L_{2}$, since they are much closer to the Earth than the Sun. Similarly, we will occasionally refer to the Earth-Moon $L_{1}$ and $L_{2}$ as the lunar or the Moon's $L_{1}$ and $L_{2}$.
} 
and other tools from dynamical systems theory (Howell, Barden, and Lo [1997]). The Solar and Heliospheric Observatory (SOHO) ${ }^{6}$ a joint project of the European Space Agency and NASA, and NASA's WIND and the Advanced Composition Explorer (ACE) are also there.

Genesis Discovery Mission. Launched in August, 2001, the Genesis Discovery Mission spacecraft swept up specks of the Sun - individual atoms of the solar windon five collector arrays the size of bicycle tires and in an ion concentrator. The goal was to collect solar wind samples and return them safely to the Earth for study into the origins of the solar system. Genesis returned its solar wind cargo to Earth via a sample-return capsule which returned to Earth in September, 2004 (see Lo, Williams, Bollman, Han, Hahn, Bell, Hirst, Corwin, Hong, Howell, Barden, and Wilson [2001]). ${ }^{7}$ The sample was the only extraterrestrial material brought back to Earth from deep space since the last of the Apollo landings in 1972, and the first to be collected from beyond the Moon's orbit.

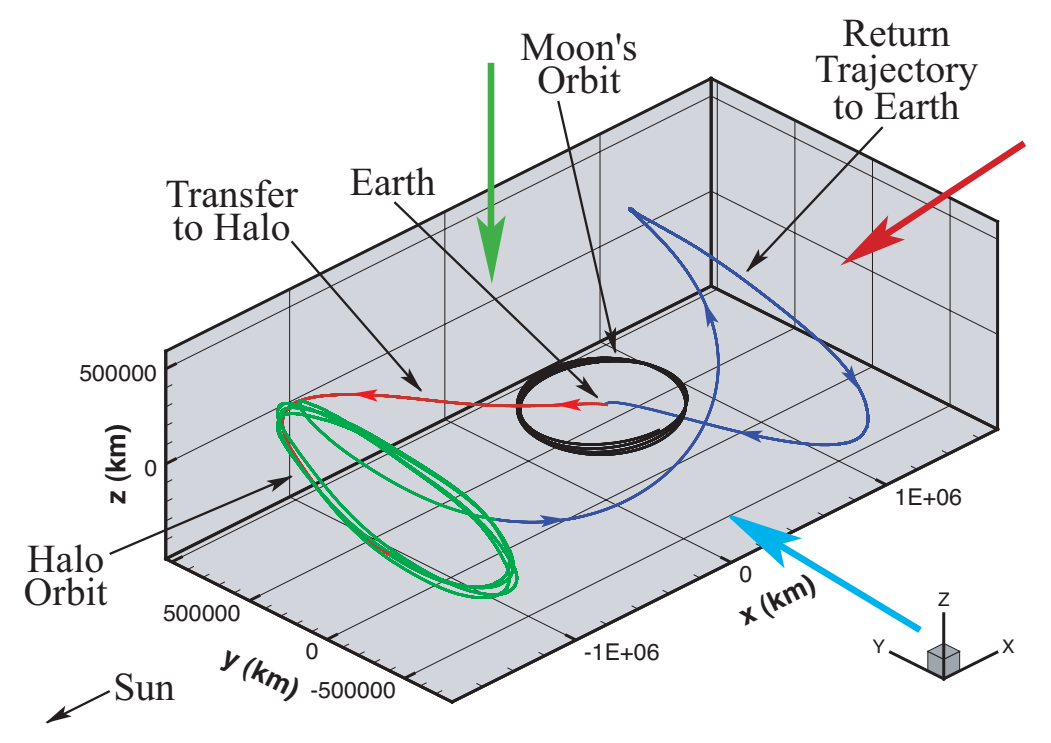

Figure 2.8. The Genesis Discovery Mission trajectory. The three arrows correspond to the three projections shown in Figure 2.9. Reprinted from Serban et al. [2002], with permission from Elsevier.

A reason Genesis was feasible as a mission is that it was designed using low energy passageways. Figure 2.8 shows a three-dimensional view of the Genesis trajectory (kindly supplied by Roby Wilson). The spacecraft was launched to a

\footnotetext{
${ }^{6} \mathrm{SOHO}$ is a spacecraft mission designed to study the internal structure of the Sun, its extensive outer atmosphere and the origin of the solar wind, the stream of highly ionized gas that blows continuously outward through the solar system. It is a joint project of ESA and NASA. See http://soho.estec.esa.nl for more information.

${ }^{7}$ See http://genesismission.jpl.nasa.gov/ for further information.
} 


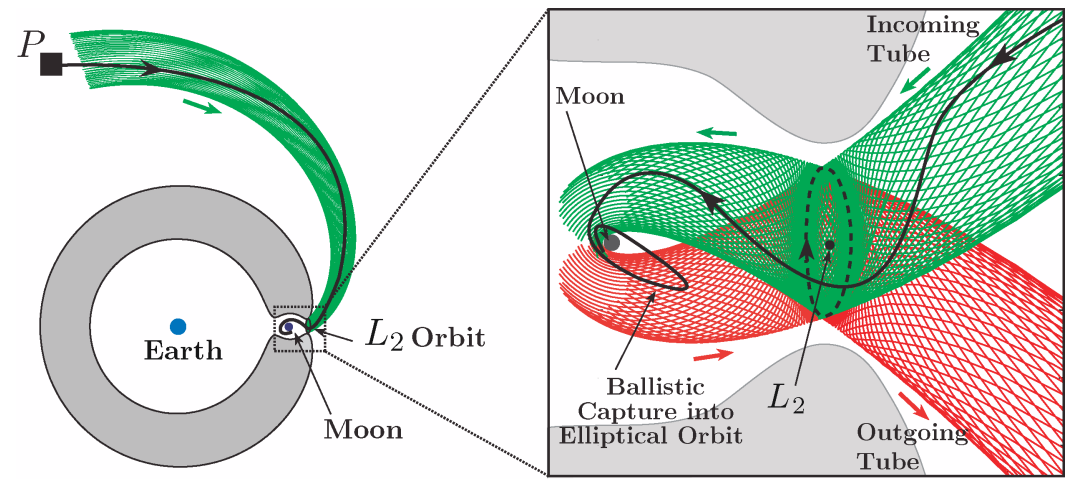

Figure 2.4. Tube leading to ballistic capture around the Moon (seen in rotating frame).

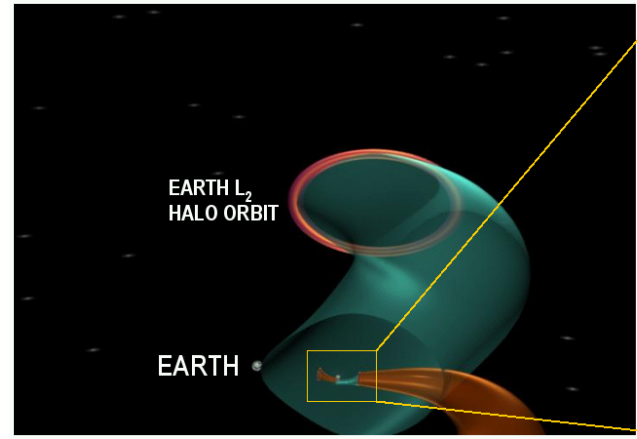

(a)

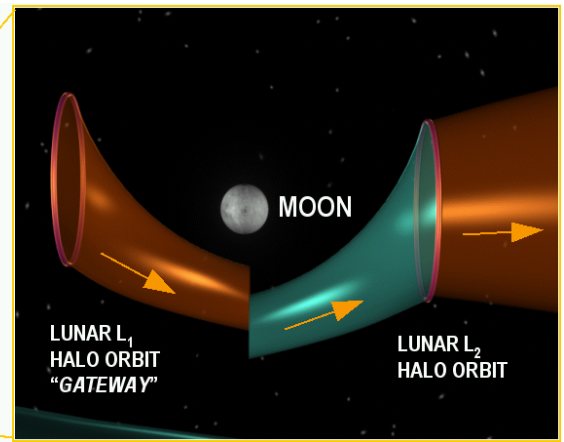

(b)

Figure 2.10. (a) The fortuitous arrangement of low energy passageways in near-Earth space implies that lunar $L_{1}$ and $L_{2}$ halo orbits are connected to halo orbits around Earth's $L_{1}$ or $L_{2}$ via low energy pathways. Many of NASA's future space telescopes located around the Earth's $L_{1}$ or $L_{2}$ may be built in a lunar $L_{1}$ orbit and conveyed to the final destination with minimal fuel requirements. (b) Shown in this close-up are two halo orbits at the lunar $L_{1}$ and $L_{2}$, respectively, and the set of invariant manifolds that provide the low energy departures from the lunar $L_{1}$ orbit. Created by Cici Koenig. First published in Lo and Ross [2001]. 


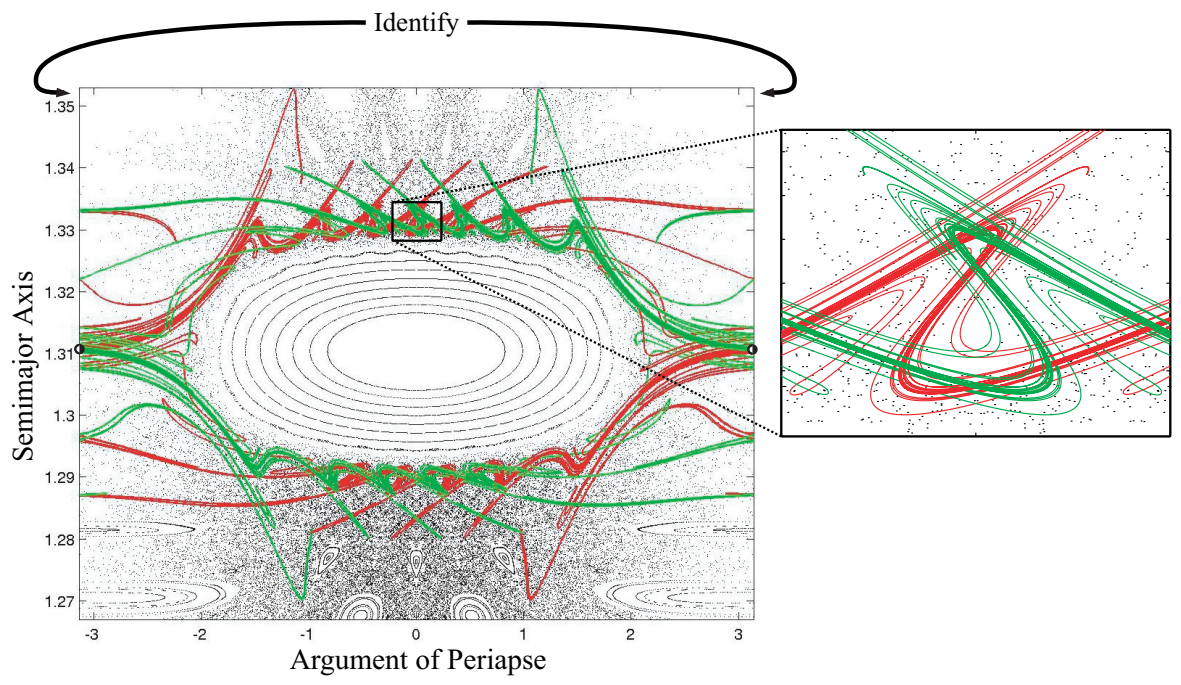

Figure 3.3. Unstable and stable manifolds comprising a homoclinic tangle that bounds a resonant region.

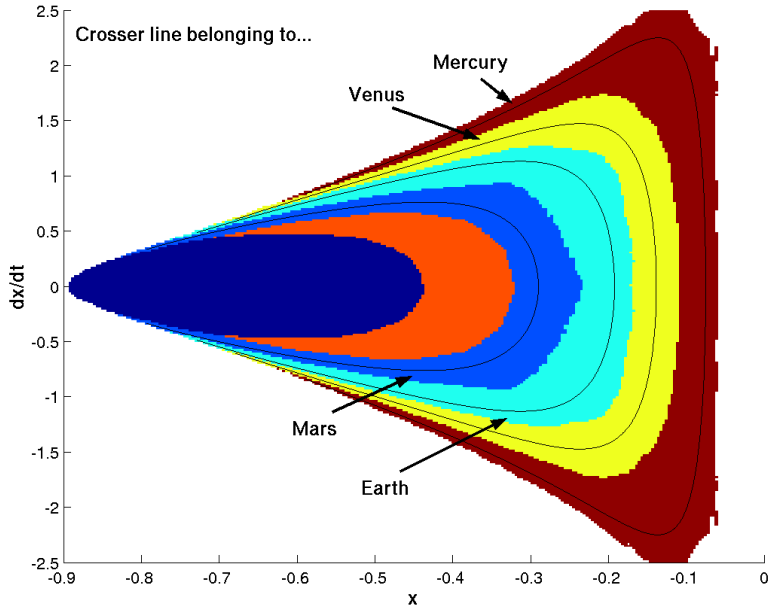

Figure 3.10. Crosser lines of all the inner planets superimposed on almost invariant sets for the Sun-Jupiter-third body system. Figure taken from Dellnitz et al. [2005b]. Courtesy of Bianca Thiere. 

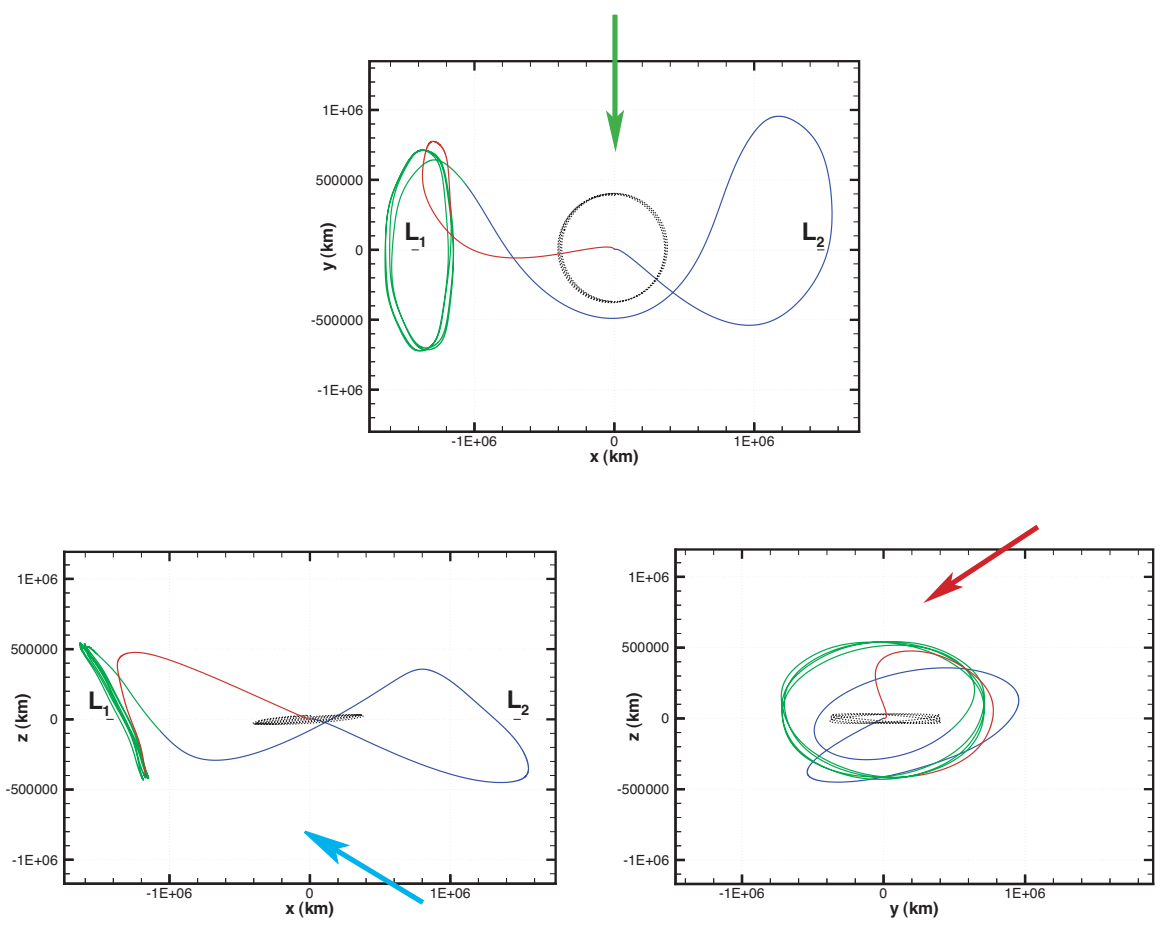

Figure 2.9. The $x y, x z$, and $y z$ projections of the three-dimensional Genesis trajectory shown in Figure 2.8.

halo orbit in the vicinity of the Sun-Earth $L_{1}$ and uses a "heteroclinic-like return" in the three-body dynamics to return to Earth. ${ }^{8}$

As noted above, $L_{1}$ is the unstable equilibrium point between the Sun and the Earth at roughly 1.5 million km from the Earth in the direction of the Sun. Genesis took a low energy path to its halo orbit, stayed there collecting samples for about 2 years, and returned home on another low energy path.

Figure 2.9 shows three orthographic projections of the Genesis trajectory. These figures, plotted in a rotating frame, show the key parts of the trajectory: the transfer to the halo, the halo orbit itself, and the return to Earth. The rotating frame is defined by fixing the $x$-axis along the Sun-Earth line, the $z$-axis in the direction normal to the ecliptic, and with the $y$-axis completing a right-handed coordinate system. The $y$-amplitude of the Genesis orbit, which extends from the $x$-axis to the maximum $y$-value of the orbit, is about 780,000 km (see Figures 2.8 and 2.9). Note that this is bigger than the radius of the orbit of the Moon, which is about $380,000 \mathrm{~km}$.

As Figures 2.8 and 2.9 show, the trajectory travels between neighborhoods of $L_{1}$ and $L_{2} ; L_{2}$ is roughly 1.5 million $\mathrm{km}$ on the opposite side of the Earth from

\footnotetext{
${ }^{8}$ The orbit is called a "halo orbit" because, as seen from Earth, the flight path follows a halo around the Sun. Such orbits were originally named for lunar halo orbits by Farquhar [1968]. By the way, setting a spacecraft exactly to the $L_{1}$ point is not a good idea, as the spacecraft's radio signals would be lost in the Sun's glare.
} 
the Sun. In dynamical systems theory, this is closely related to the existence of a heteroclinic connection between the $L_{1}$ and $L_{2}$ regions.

The deeper dynamical significance of the heteroclinic connection for the planar three-body problem is that it allows a classification and a construction of orbits using symbolic dynamics, as was shown in Koon, Lo, Marsden, and Ross [2000], and similar phenomena are seen when the third degree of freedom is included, as discussed in Gómez et al. [2004].

One of the attractive and interesting features of the Genesis trajectory design is that the three-year mission, from launch all the way back to Earth return, requires no deterministic maneuver whatsoever and automatically injects into the halo orbit.

It is difficult to use traditional classical algorithms ${ }^{9}$ to find a near-optimal solution like that of Genesis, so the design of such a low energy trajectory is facilitated by using dynamical systems methods. This is achieved by using the stable and unstable manifolds as guides in determining the end-to-end trajectory. That Genesis performs its huge exotic trajectory using a deterministic $\Delta V$ of zero (i.e., no fuel) has created a great deal of interest in both the astronautical and mathematical communities.

Lunar $L_{1}$ Gateway Station. The work on Genesis has inspired deeper exploration of the dynamics in Earth's neighborhood (see Lo and Ross [2001]). NASA desires to develop a robust and flexible capability to visit several potential destinations, as suggested by the metro map, Figure 2.7. A Lunar Gateway Station in the vicinity of the lunar $L_{1}$ libration point (between the Earth and the Moon) was proposed as a way station for transfers into the solar system and into the EarthSun halo orbits. This is enabled by a historical accident: the energy levels of the Sun-Earth $L_{1}$ and $L_{2}$ points differ from those of the Earth-Moon system by only $50 \mathrm{~m} / \mathrm{s}$ (as measured by maneuver velocity). The significance of this coincidence to the development of space cannot be overstated. For example, this implies that the lunar $L_{1}$ halo orbits are connected to halo orbits around Earth's $L_{1}$ and $L_{2}$ via low energy pathways, as illustrated in Figure 2.10.

Many of NASA's future space observatories located around the Earth's $L_{1}$ or $L_{2}$ points may be built in a lunar $L_{1}$ orbit and conveyed to the final destination with minimal propulsion requirements. When the spacecraft or instruments require servicing, they may be returned from Earth libration orbits to the lunar $L_{1}$ orbit, where human servicing may be performed, which was shown to be of vital importance for keeping the Hubble Space Telescope operable. Since a lunar $L_{1}$ orbit may be reached from Earth in only three days, the infrastructure and complexity of long-term space travel is greatly mitigated. The same orbit could reach any point on the surface of the Moon within hours, making it a perfect location for the return of humans to the Moon. A lunar $L_{1}$ orbit is also an excellent point of departure and arrival for interplanetary flights to Mars, the asteroids, and the outer solar system. Several lunar and Earth encounters may be added to further reduce the launch cost and open up the launch period. A lunar $L_{1}$ is therefore a versatile hub for a space transportation system.

Multi-Moon Orbiters. Using low energy passageways is in no way limited to the inner solar system. For example, consider a spacecraft in the gravity field of Jupiter and its planet-sized moons. A possible new class of missions to the outer

\footnotetext{
${ }^{9}$ See, for example, Farquhar and Dunham [1981], Farquhar, Muhonen, Newman, and Heuberger [1980], and Farquhar, Muhonen, and Richardson [1977].
} 
planet moon systems has been proposed (Koon, Lo, Marsden, and Ross [1999], Ross, Koon, Lo, and Marsden [2003]). These are missions in which a single spacecraft orbits several moons of Jupiter (or any of the outer planets), allowing long duration observations. Using this multi-moon orbiter approach, a single scientific spacecraft orbits several moons of Jupiter (or any of the outer planets) for any desired duration, allowing long duration observations instead of flybys lasting only seconds. For example, a multi-moon orbiter could orbit each of the galilean moons - Callisto, Ganymede, Europa, and Io - one after the other, using a technologically feasible amount of fuel. This approach should work well with existing techniques, enhancing trajectory design capabilities for missions such as a mission to assess the possibility of life on Jupiter's icy moons.

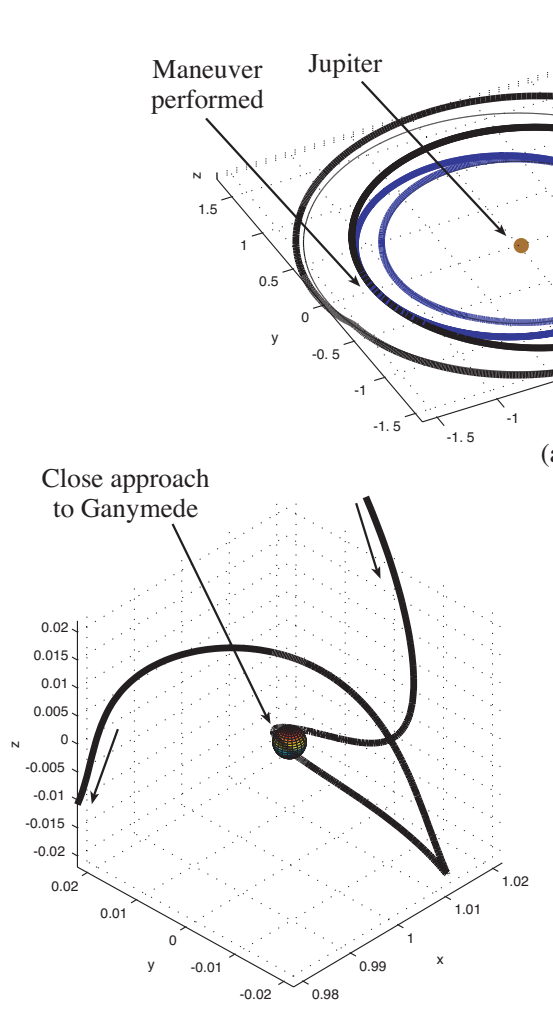

(b) (a) a)

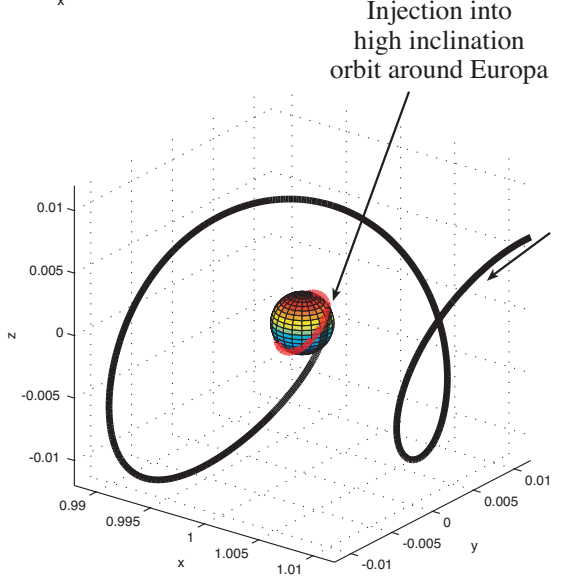

(c)

Figure 2.11. A multi-moon orbiter space mission concept for the Jovian moons. (a) We show a spacecraft trajectory coming into the Jupiter system and transferring from Ganymede to Europa using a single impulsive maneuver, shown in a Jupiter-centered inertial frame. (b) The spacecraft performs one loop around Ganymede, using no propulsion at all, as shown here in the Jupiter-Ganymede rotating frame. (c) The spacecraft arrives in Europa's vicinity at the end of its journey and performs a final propulsion maneuver to get into a high inclination circular orbit around Europa, as shown here in the Jupiter-Europa rotating frame. 


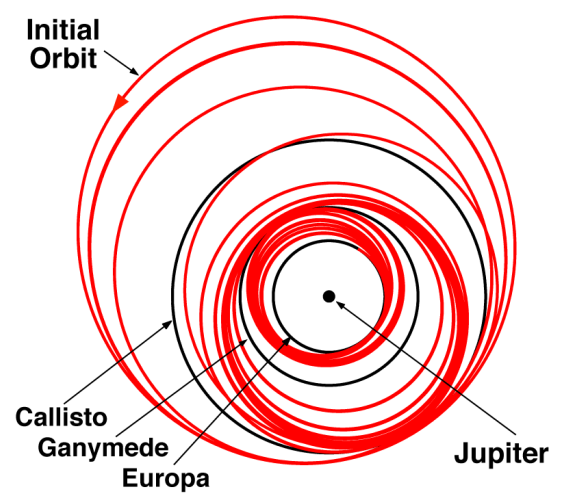

FIGURE 2.12. The trajectory shown is a simulation of a restricted 5 -body problem and requires a vanishingly small $\Delta V$. The solution likely shadows a zero $\Delta V$ solution, i.e., a purely natural solution which may bring an asteroid interloper into intimate contact with the Jupiter system. The multi-moon orbiter is a general concept that is applicable for any multi-moon system.

Figure 2.11 shows a low energy transfer trajectory from an initial Jovian insertion trajectory to Ganymede. After one orbit around Ganymede including a close approach, the spacecraft heads onward to Europa, ending in a high inclination orbit around the icy moon.

The fuel requirements for such a tour can be made even smaller if resonant phenomena, as discussed in the next section, are incorporated. By utilizing resonant gravity assists with Jupiter's moons, in addition to ballistic capture and escape orbits leading toward or away from temporary capture orbits about a moon, a tour can be constructed using very little fuel, such as the one shown in Figure 2.12.

Optimization. As we have seen, one of the reasons that making use of the subtleties of the three-body problem to design missions is that they give more fuel efficient trajectories. In other words, they are heading in the direction of optimal trajectories.

One study in this direction was the work of Serban et al. [2002] which used the optimization software COOPT (Control and Optimization), developed in the group of Linda Petzold, to optimize the fuel needed to get the Genesis spacecraft back on track towards its intended halo (or Lissajous) orbit in case of a launch error. That paper has a brief description of this software and how it works, but basically it is a multiple shooting method that is combined with other DAE (Differential Algebraic Equation) software and SQP (Sequential Quadratic Programming) methods.

Although many of the trajectories outlined above are already very efficient, they also need to be optimized. For example, when one is transferring from one threebody system to another, what is the optimal time to do the transfer burn? What if one has a low thrust spacecraft? Such questions can be dealt with using optimization software such as COOPT, although each situation can have its own peculiarities.

Another approach to optimization, due to Junge et al. [2005], is called DMOC (Discrete Mechanics and Optimal Control) and is based on the use of discrete 
mechanics techniques together with SQP methods. Discrete mechanics methods are based on a discrete version of the variational principles of mechanics, namely Hamilton's principle for mechanical systems and the Lagrange-d'Alembert principle for mechanical systems subject to external forces.

Discrete mechanics methods have been available for quite some time, going back to discrete optimal control methods in the 1960s. These techniques are closely related to variational integration methods. These methods include symplectic integration algorithms, but also include systems with forcing and dissipation. As such, numerical evidence shows that they have a remarkable respect for the energy budget of a system, far better than any standard numerical error analysis would justify. A development, survey and history of such methods can be found in Marsden and West [2001], Lew et al. [2004].

Roughly speaking, the basic principle of mechanics for a system with configuration variables $q$ (coordinates in a configuration manifold $Q$ ) and a Lagrangian (usually the kinetic minus potential energies) $L(q, \dot{q})$ and with external forcing (controls) $F(q, \dot{q})$, namely the Lagrange-d'Alembert principle,

$$
\delta \int_{a}^{b} L(q(t), \dot{q}(t)) d t+\int_{a}^{b} F \cdot \delta q d t=0
$$

is replaced by its discrete counterpart. This is done by replacing curves $q(t)$ in the configuration manifold $Q$ by discrete sets of points $\left(q_{1}, q_{2}, \ldots, q_{N}\right)$ in $Q$, replacing the Lagrangian by a discrete Lagrangian $L_{d}$ that is a function of pairs $\left(q_{i}, q_{i+1}\right)$, and replacing the integrals by an appropriate discrete sum. Asking this discrete sum to be stationary under variations of these points (but with the first and last held fixed) leads to the discrete Lagrange-d'Alembert equations, which is a set of algebraic equations. The cost function in the optimization problem, which is normally an integral of the velocity phase space variables $(q(t), \dot{q}(t))$ along with the controls, is likewise replaced by its discrete counterpart.

One now seeks to optimize the discrete cost function subject to the discrete Lagrange-d'Alembert equations as constraints (along with any other constraints in the problem, such as actuator limits, collision constraints, etc.). This procedure discretizes the problem, and as such it can be directly handed off to SQP routines.

This relatively straightforward procedure already indicates that DMOC has the considerable advantage of simplicity as well as respecting the geometry of Lagrangian and Hamiltonian mechanics (such as the symplectic structure, conservation laws, having good energy behavior, etc.).

One of several examples that DMOC has been applied to is of interest in finding Earth-like planets in distant solar systems. This system involves the deployment of an array of interferometers that may be used to detect the chemical composition in the atmosphere of the distant planet. The NASA version of this is called TPF, Terrestrial Planet Finder, while ESA's version is called DARWIN. See Figure 2.13.

In this example, ${ }^{10}$ a group of six spacecraft in the vicinity of a Halo-orbit are required to adopt a planar hexagonal formation with center on the Halo orbit and to point in a given direction. The optimization problem is to have the spacecraft get to the final formation from a given initial configuration in such a way that a measure of the fuel consumed is minimized (one could also use a weighted sum of fuel consumed and the time required). Here DMOC gives a solution as indicated in

${ }^{10}$ We are grateful to Oliver Junge and Sina Ober-Blöbaum for providing this example. 


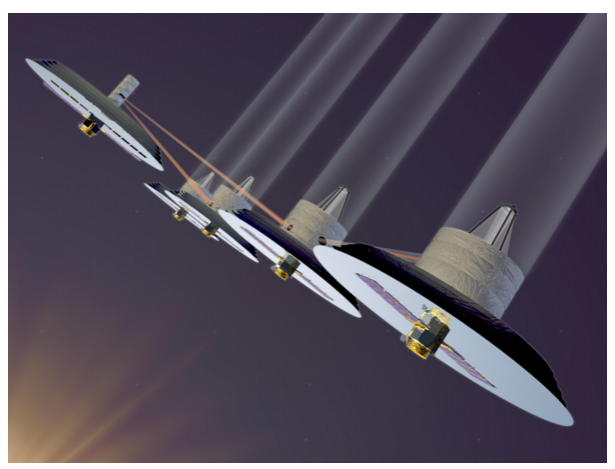

Figure 2.13. A system of spacecraft can act as a long baseline set of interferometers capable of detecting Earth-like planets in distant solar systems. Source: NASA.

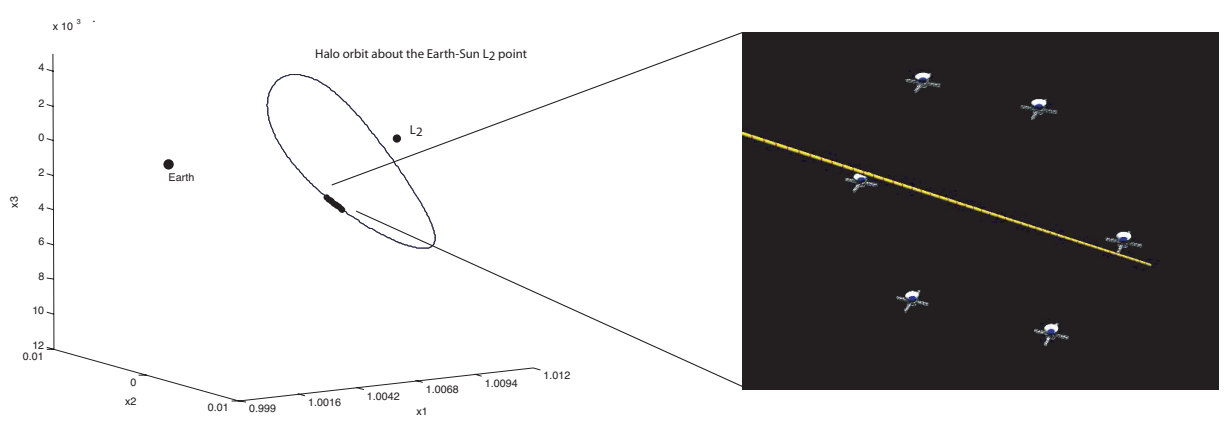

Figure 2.14. Using DMOC to deploy a group of six spacecraft that are in a halo orbit around $L_{2}$ to optimally assume a hexagonal pattern and point in a given direction.

Figure 2.14; the attitude dynamics of each spacecraft, as well as collision avoidance, is taken into account.

Because of the good respect for the energy budget of a system, as well as its computational simplicity, one can expect that DMOC will be useful for low thrust missions as well as in formation studies.

\section{TRANSPORT IN THE SOLAR SYSTEM AND BEYOND}

As we have indicated, there are many phenomena in the solar system that involve interesting transport processes. Examples include the transport of Mars rocks to Earth (the rocks could be thrown into Mars orbit by a meteor impact, for instance) and the transport of asteroids and comets from outside of Jupiter's orbit to inside of Jupiter's orbit through the two Jupiter necks shown in Figure 2.3. Several comets, such as Oterma, did just that (this is described in Koon, Lo, Marsden, and Ross [2000]).

For such processes, one can ask, "What is the transport rate?" More specifically, we might ask, "how does one compute the percentage of a random distribution on an appropriate energy shell after 1000 years that will go from outside of Jupiter's 
orbit (the exterior realm) to inside (the Sun, or interior, realm)?" Similarly, "what is the probability of transport of Kuiper belt objects from outside of Neptune's orbit to inside?"

To study such questions, we need a few more tools from theoretical and computational dynamical systems.

Poincaré Sections. For the planar three-body problem, the energy surfaces are three dimensional. Thus, using a Poincaré surface of section at fixed energy $E$ represents the system as a two-dimensional area-preserving map. For example, in the Sun-Jupiter-third body system, we might choose a section in the interior realm, as shown in Figure 3.1.

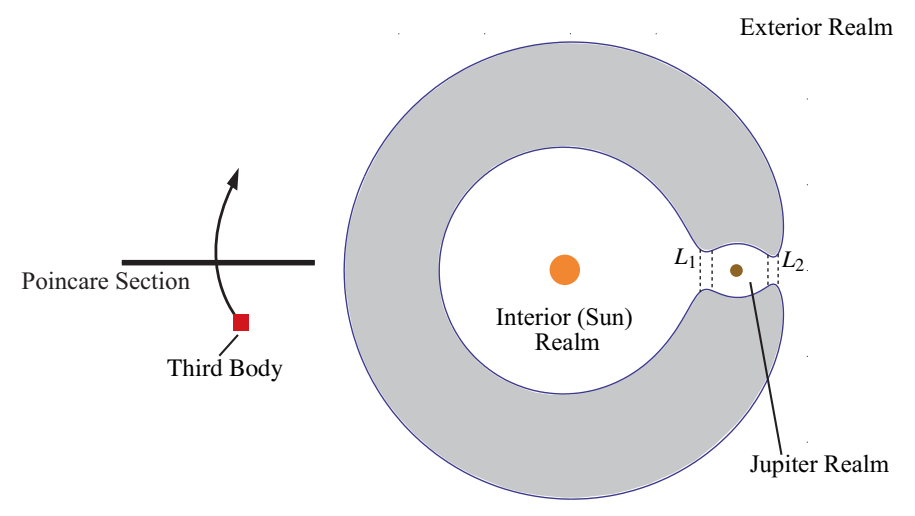

Figure 3.1. A Poincaré surface of section in the exterior realm of the Sun-Jupiter-third body system.

Such a procedure then produces a standard Poincaré map picture, as shown in Figure 3.2.

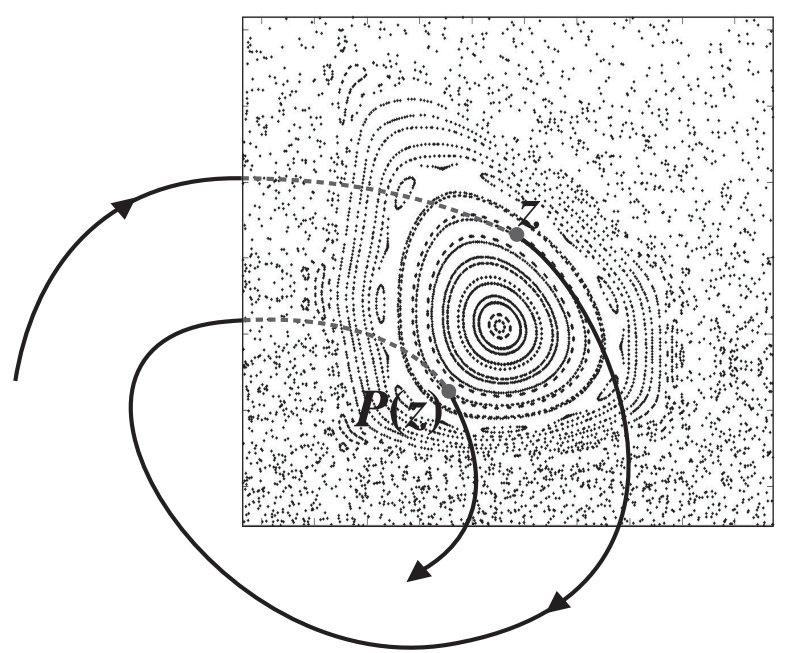

Figure 3.2. A Poincaré map produced by intersecting orbits with a Poincaré section. 
As we have indicated - and it is also important for transport in both the celestial as well as the molecular context - these different Poincaré sections are linked by the Conley-McGehee tubes.

MANGEN. To carry out the needed computations, software is of course required. While there are lots of packages available, we shall concentrate on two of them. First of all, MANGEN (Manifold Generation) computes, amongst many other things, invariant manifolds and transport rates between different resonant regions using dynamical systems methods such as lobe dynamics (see Rom-Kedar and Wiggins [1990], Meiss [1992], Wiggins [1992], Rom-Kedar [1999]). While this software was originally developed for the study of fluid systems (see Lekien [2003]), it has proved to be useful for astrodynamics as well as in molecular systems! Mathematics of course provides the common structures in the three areas that enable this.

A sample computation of invariant manifolds in a Poincaré section using MANGEN in the astrodynamics area is shown in Figure 3.3.

One of the interesting questions in dynamical astronomy is to ask about the transport between various regions. Some resonant regions, such at the one shown in Figure 3.4 for a region outside the orbit of Jupiter, have "leaky" boundaries due to homoclinic tangles. Using lobe dynamics, MANGEN can compute the transport between such regions and neighboring regions.

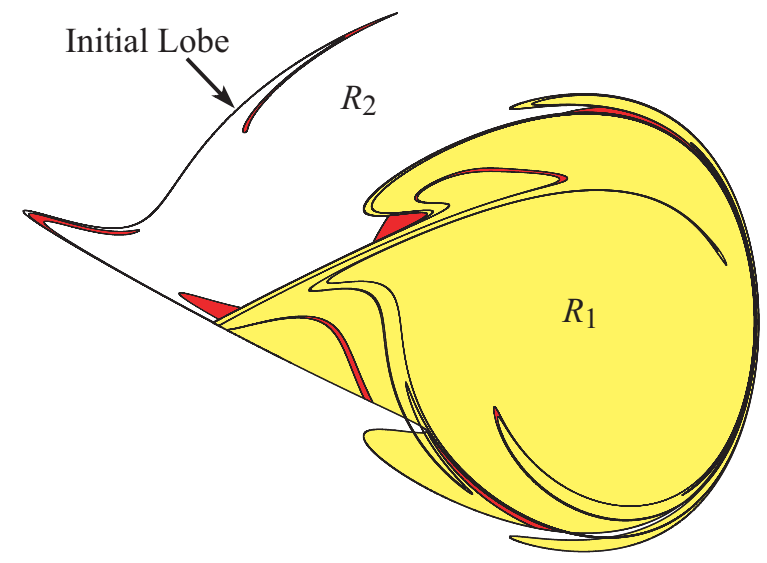

FiguRE 3.4. MANGEN can compute the transport rate between these two resonant regions $R_{1}$ and $R_{2}$ in the Sun-Jupiter-third body system. One lobe beginning in $R_{2}$ (upper left) and several of its images under the Poincaré map are shown. The axes in this Poincaré section are $(x, \dot{x})$.

GAIO. A second piece of software that is very useful is GAIO (Global Analysis of Invariant Objects); see Dellnitz et al. [2001] and Dellnitz and Junge [2002]. It uses a set-oriented methodology, taking a global point of view to compute sets of dynamical interest rather than focusing on individual trajectories. The idea is to cover the region of phase space with boxes and to refine using box subdivisions. A given dynamical system is discretized using a map and then an associated graph is constructed. The nodes are the box centers, and edges connect those nodes that are dynamically related. This set up allows one to make use of techniques from graph theory, such as graph partitioning. 
One of the key concepts in this area is the notion of an almost invariant set (AIS), which corresponds to a set containing relatively long-lived dynamical trajectories. An example of an AIS are the above resonance regions. This notion is also important in, for example, biomolecules, where it corresponds to molecular conformations.

There are two related ways to compute almost invariant sets. The first is to use graph partitioning software such as PARTY (Monien, Preis and Diekmann [2000]). The idea is to find efficient ways to cut the graph so that the traffic flow (that is, the transport rate) across the cut is minimized. A second method is the use of eigenfunctions of the associated Perron-Frobenius operator (the induced map on measures). We refer to Dellnitz and Junge [2002] and to Dellnitz et al. [2005] for a survey of these methods and for further references. In either case, the computation

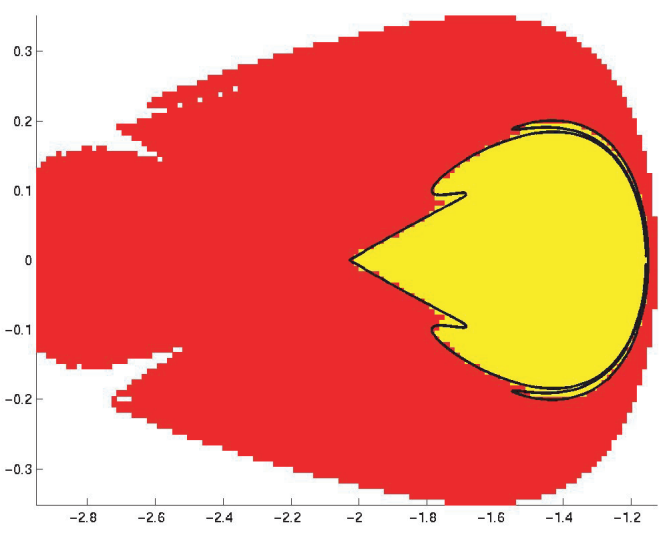

FiguRE 3.5. Resonance region for the three-body Sun Jupiter system computed using GAIO.

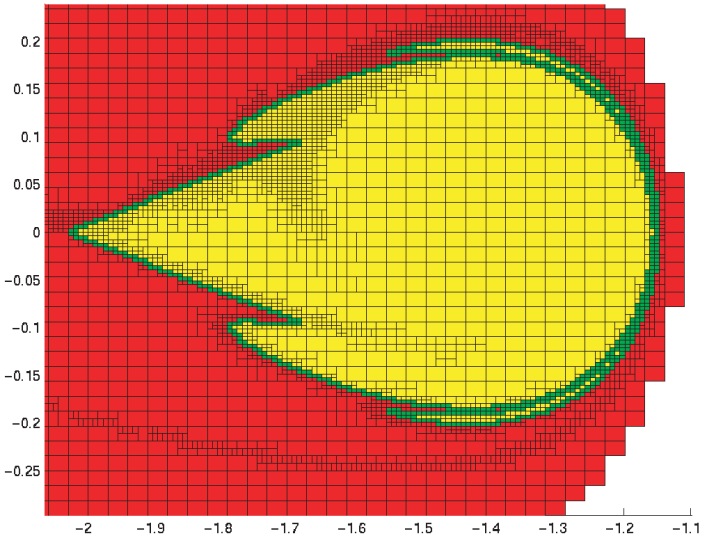

FIGURE 3.6. GAIO and MANGEN working together. 
of transport rates between two AISs is naturally computed within these set oriented methods.

Figure 3.5 shows the same resonance region as above, but computed using GAIO. In some circumstances, such as shown in Figure 3.6, GAIO and MANGEN can work together to produce more efficient adaptive algorithms.

Using these techniques, one gets very concrete answers for transport rates. For example, it is shown in Dellnitz et al. [2005] that there is a $28 \%$ probability that a randomly chosen particle will go from region $R_{1}$ to $R_{2}$ in 1,817 Earth years.

Mars Crossing Asteroids. Figure 3.7 shows the data from the Hilda group of asteroids and comets that lie between Jupiter and Mars in a belt relatively close to Jupiter.

Figure 3.8 shows a Poincaré map for a cut chosen at an energy level appropriate to the Hilda group and cutting across it. The coordinates are $x$ along the cut and $\dot{x}$ the corresponding velocity. Also shown is the Mars crosser line, i.e., the coordinates $(x, \dot{x})$ of points whose Keplerian orbit with that initial condition will just graze the orbit of Mars. Hildas become Mars-crossing asteroids by going from the left of this curve, where they usually reside, to the right of it.

A subset of the Hilda group is the quasi-Hildas, objects known to receive strong perturbations from Jupiter (Kresák [1979], Ross [2003]). As shown in Dellnitz et al. [2005a], GAIO can locate the quasi-Hilda region as one of the AISs in this threebody problem. Drawing in the set corresponding to the Mars crosser line, as shown in Figure 3.9, we can then ask, "What is the probability that, in a certain period of time, an object in the quasi-Hilda region becomes a Mars-crossing asteroid?" GAIO gives rather concrete answers. It shows that the probability for a typical particle to leave the quasi-Hilda region is around $6 \%$ after 200 iterates of the map,

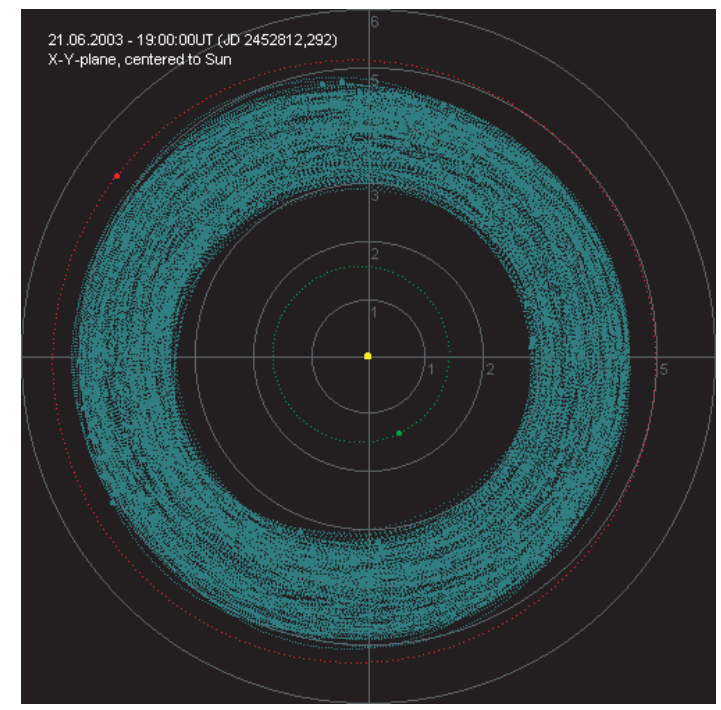

FiguRE 3.7. Orbits of asteroids in the Hilda group. Jupiter is the large dot on the outer dotted circle and Mars is the large dot on the inner dotted circle. Figure courtesy of Alcyone Software, Germany, http://www. alcyone-software.com. 


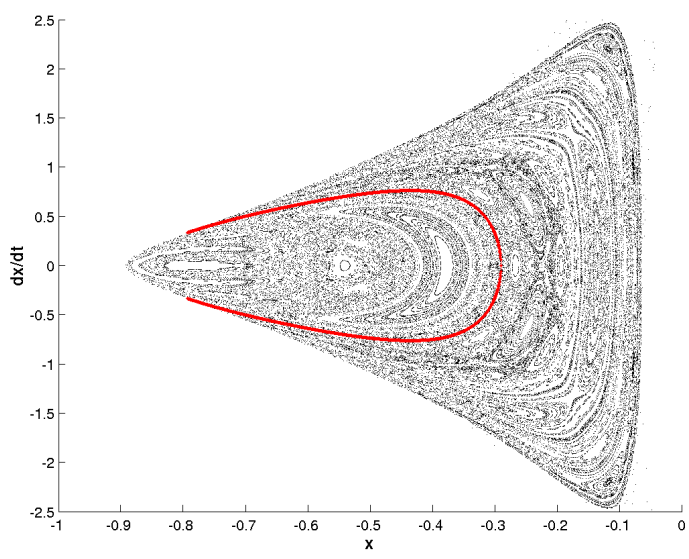

Figure 3.8. A Poincaré map corresponding to a section cutting across the Hilda group. Reprinted with permission from Dellnitz et al. [2005a]. Copyright 2005 by the American Physical Society.

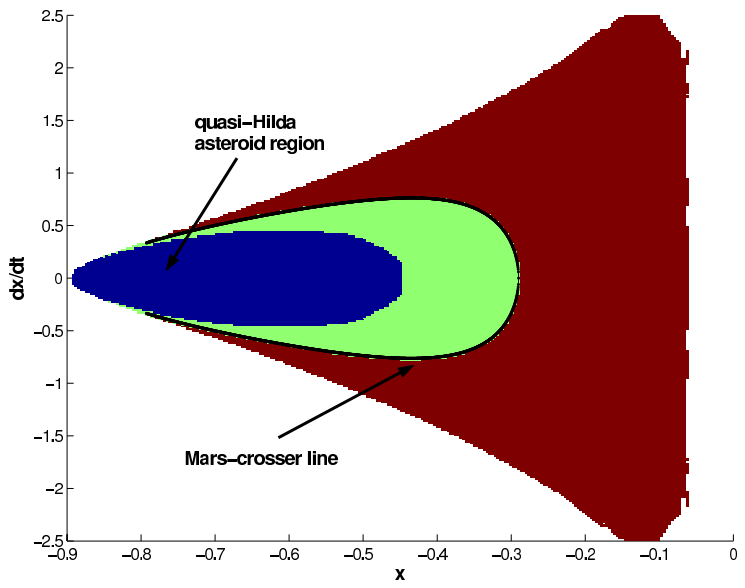

Figure 3.9. The quasi Hilda set (an AIS) and the Mars crosser set. Reprinted with permission from Dellnitz et al. [2005a]. Copyright 2005 by the American Physical Society.

which corresponds to a transit time between 2000 and 6000 Earth years, depending on the location of the particle within the quasi-Hilda region.

But there is much more to the story, as shown in Figure 3.10, taken from Dellnitz et al. [2005b]. This figure shows all of the inner planet crosser curves; notice how they lie in the middle of various of the Sun-Jupiter-third body almost invariant sets (as computed by GAIO)! The missing curve in the region between Mars and Jupiter is where the main asteroid belt lies. Since almost invariant sets, by their nature, are places where material in the Sun-Jupiter-third body system lingers for a long time, one can speculate that material should accumulate in these locations (assuming of course that a primordial Jupiter was formed first). A possible scenario 
is that material was streaming from a cloud in the primordial Jupiter realm, leaking out of Jupiter's Lagrange bottlenecks, and some of this material migrated under encounters with Jupiter to the terrestrial planet region.

The almost invariant sets are computed just with the Sun-Jupiter-third body system, which in principle is independent of the knowledge of the location of the other planets. The figure clearly shows that these are not independent at all, and it suggests that the Jupiter system in fact drove the formation of the whole solar system. This computation is consistent with the Titius-Bode Law for the spacing of the planets (but does not "explain it") and could be repeated for any other planetary system. Of course additional analysis and simulation are needed to make this definitive, and this is planned for future research. For example, preliminary computations show that this result is not affected if Saturn is added to the SunJupiter system. It is worth noting that important questions could be addressed using the almost invariant sets, beyond simply the spacing of the planets. For instance, what would this Jupiter-driven formation scenario imply, if anything, about the relative masses of the terrestrial planets? Furthermore, is there any dynamical connection between the terrestrial planets and the rocky, planet-sized moons of Jupiter?

The above result highlights one example of the many miracles hidden in the solar system and the relations between the planets! One might even be hopeful that such ideas will be useful in the search for Earth-like planets in other solar systems.

Tubes on Galactic Scales. Tubes are known to govern structure and motion even over galactic scales (Fukushige et al. [2000]; Ross [2005]). The huge tails emanating out of some star clusters in orbit about our galaxy are due to stars slipping into tubes connecting the star cluster with the space outside. This is shown in the left panel of Figure 3.11, from a large $N$-body simulation of Combes et al. [1999], where
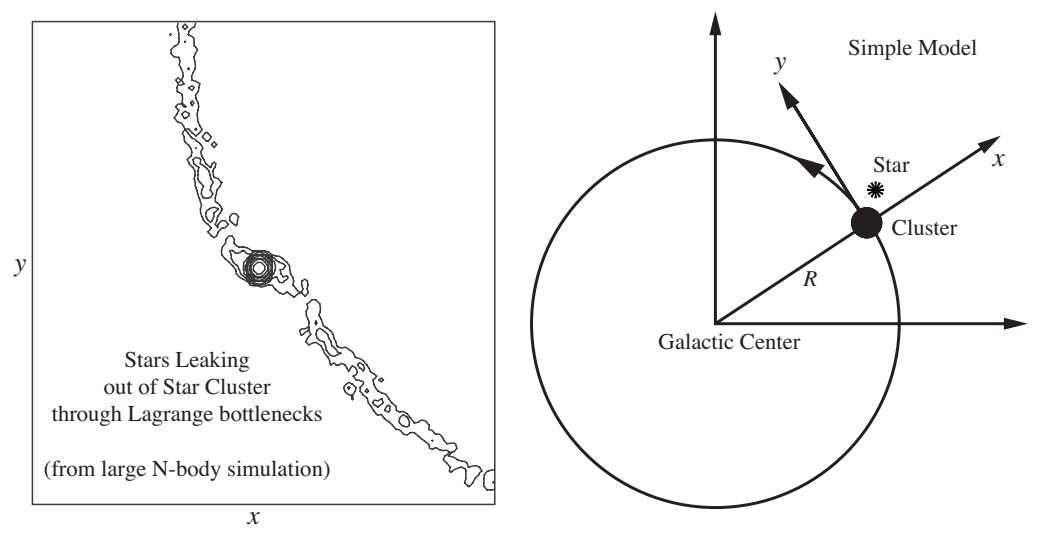

Figure 3.11. Large $N$-body simulations of star cluster 'evaporation' reveal characteristic tube features (left) out of which stars escape. The time scale of evaporation can be estimated in a simple model (right) using the tube structures associated with Lagrange points on either side of the cluster, similar to those described in the three-body problem. Left-hand side of figure first published in Combes et al. [1999]. Reprinted with permission. 


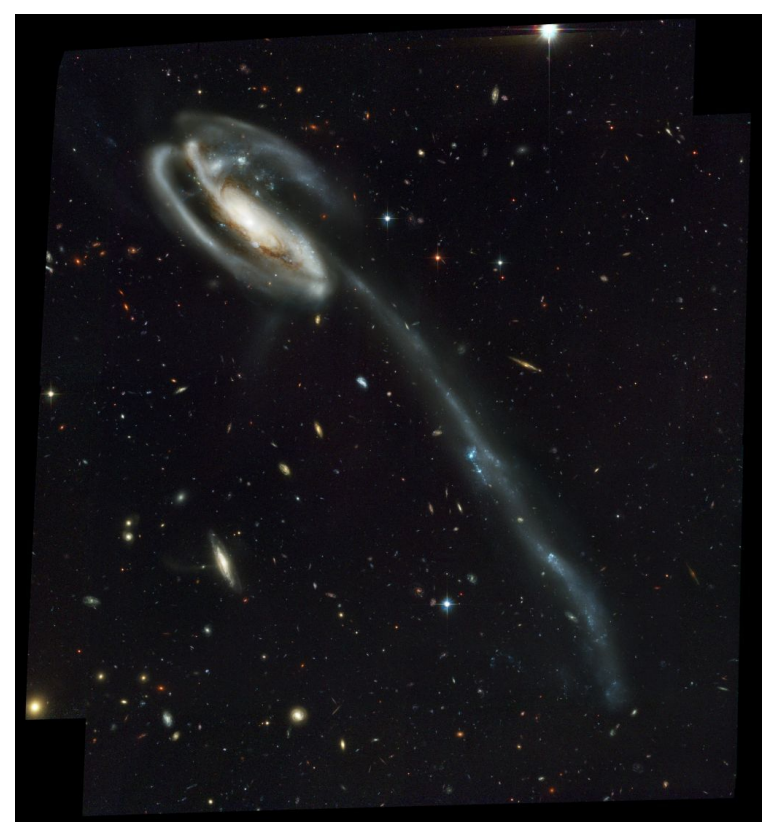

Figure 3.12. The Tadpole Galaxy's tidal tail. Source: ACS Science \& Engineering Team, NASA.

$N$ is on the order of a million. It is believed that this escape process can eventually lead to the 'evaporation' of some star clusters over tens of billions of years.

The estimation of this evaporation time scale is possible using a very simple model that is in principle similar to the three-body model. Shown schematically in the right panel of Figure 3.11, the star cluster is modelled as a smooth potential (due to the cluster stars) plus the steady tidal field of the galaxy. Stars which are above the energy of the Lagrange points escape via Conley-McGehee tubes. From the tubes the evaporation time scale, measured as the time for the cluster to drop from $N$ to $N / 2$ stars, can be determined in a similar way to the transport calculations described above.

Tidal tails are not restricted to star clusters alone. Figure 3.12 shows the disrupted spiral galaxy Arp 188, the Tadpole Galaxy. One possible scenario is that a more compact intruder galaxy crossed in front of the Tadpole. During the close encounter, tidal forces drew out the Tadpole's stars, gas, and dust, forming the spectacular tail, which is 280 thousand light-years long.

\section{ACKNOWLEDGEMENTS}

We acknowledge the help and advice of our collaborators mentioned in the abstract as well as the assistance of Wendy McKay.

\section{ABOUt THE AUTHORS}

Jerrold E. Marsden is the Carl F. Braun Professor of Engineering and Control and Dynamical Systems at Caltech. He is the recipient of the 1990 AMS-SIAM 
Norbert Wiener Prize, a 2000 Max Planck Research Award, and the 2005 SIAM John von Neumann Award.

Shane Ross is currently at the University of Southern California, where he holds an NSF Mathematical Sciences Postdoctoral Fellowship. He has received awards from NASA and the American Astronautical Society for his work on astrodynamics.

\section{REFERENCES}

Bate, R. R., D. D. Mueller, and J. E. White [1971], Fundamentals of Astrodynamics. Dover, New York.

Belbruno, E. [2004], Capture Dynamics and Chaotic Motions in Celestial Mechanics: With Applications to the Construction of Low Energy Transfers, Princeton University Press. MR2029316 (2004m:70020)

Combes, F., S. Leon, and G. Meylan [1999], N-body simulations of globular cluster tides, Astron. Astrophys. 352, 149-162.

Dellnitz, M., G. Froyland, and O. Junge [2001], The algorithms behind GAIO-set oriented numerical methods for dynamical systems. In Ergodic theory, analysis, and efficient simulation of dynamical systems, pages 145-807. Springer, Berlin. MR1850305 (2002k:65217)

Dellnitz, M., K. Grubits, J. E. Marsden, K. Padberg, and B. Thiere [2005], Setoriented computation of transport rates in 3-degree of freedom systems: the Rydberg atom in crossed fields, Regular and Chaotic Dynamics 10, 173-192.

Dellnitz, M. and O. Junge [2002], Set oriented numerical methods for dynamical systems. In Handbook of Dynamical Systems, Vol. 2, pages 221-264. North-Holland, Amsterdam. MR1900656 (2003f:37161)

Dellnitz, M., O. Junge, W. S. Koon, F. Lekien, M. W. Lo, J. E. Marsden, K. Padberg, R. Preis, S. Ross, and B. Thiere [2005], Transport in dynamical astronomy and multibody problems, Intern. J. of Bifurcation and Chaos 15, 699-727. MR2136742

Dellnitz, M., O. Junge, M. W. Lo, J. E. Marsden, K. Padberg, R. Preis, S. Ross, and B. Thiere [2005a], Transport of Mars-crossing asteroids from the quasi-Hilda region, Physical Review Letters 94, 231102-1-231102-4.

Dellnitz, M., O. Junge, J. E. Marsden, K. Padberg, R. Preis, S. Ross, and B. Thiere [2005b], Almost invariant sets and celestial mechanics (in preparation).

Dunn, G. L. [1962], A high-speed data link for farside lunar communications, General Electric Co. Report 62 SPC-5, March 1962.

Farquhar, R. W. [1966], Station-Keeping in the Vicinity of Collinear Libration Points with an Application to a Lunar Communications Problem. In Space Flight Mechanics, Science and Technology Series, volume 11, pages 519-535. American Astronautical Society, New York.

Farquhar, R. W. and D. W. Dunham [1981], A new trajectory concept for exploring the Earth's geomagnetic tail, Journal of Guidance and Control, 4, 192-196.

Farquhar, R. W., D. P. Muhonen, C. Newman, and H. Heuberger [1980], Trajectories and Orbital Maneuvers for the First Libration-Point Satellite, Journal of Guidance and Control, 3, 549-554.

Farquhar, R. W., D. P. Muhonen, and D. L. Richardson [1977], Mission Design for a Halo Orbiter of the Earth, Journal of Spacecraft and Rockets 14, 170-177.

Farquhar, R. [1968], The Control and Use of Libration-Point Satellites, PhD thesis, Stanford University. 
Fukushige, T. and D. C. Heggie [2000], The time-scale of escape from star clusters, Mon. Not. R. Astron. Soc. 318, 753-761.

Gabern, F., W.-S. Koon, J. E. Marsden and S. D. Ross [2005], Theory and computation of non-RRKM lifetime distributions and rates in chemical systems with three or more degrees of freedom, Physica $D$ (to appear).

Gómez, G., W. S. Koon, M. W. Lo, J. E. Marsden, J. Masdemont, and S. D. Ross [2004], Connecting orbits and invariant manifolds in the spatial restricted three-body problem, Nonlinearity 17, 1571-1606. MR2086140 (Review)

Howell, K., B. Barden, and M. Lo [1997], Application of dynamical systems theory to trajectory design for a libration point mission, The Journal of the Astronautical Sciences 45, 161-178. MR1604793

Jaffé, C., S. D. Ross, M. W. Lo, J. E. Marsden, D. Farrelly, and T. Uzer [2002], Statistical Theory of Asteroid Escape Rates, Phys. Rev. Lett. 89, 011101-1.

Junge, O., J. E. Marsden, and S. Ober-Blöbaum [2005], Discrete mechanics and optimal control, IFAC Proceedings (to appear).

Koon, W. S., M. W. Lo, J. E. Marsden, and S. D. Ross [1999], Constructing a Low Energy Transfer between Jovian Moons. In Celestial Mechanics : an international conference on celestial mechanics, Evanston, Illinois.

Koon, W. S., M. Lo, J. E. Marsden, and S. Ross [2000], Heteroclinic connections between periodic orbits and resonance transitions in celestial mechanics, Chaos 10, 427-469. MR1765636 (2001f:70009)

Koon, W. S., M. Lo, J. E. Marsden, and S. Ross [2005], Dynamical Systems, the Three-Body Problem and Space Mission Design (to be published).

Kresák, L. [1979], Dynamical interrelations among comets and asteroids, in Asteroids, Univ. of Arizona Press, Tucson, 289-309.

Lekien, F. [2003], Time-Dependent Dynamical Systems and Geophysical Flows, PhD thesis, California Institute of Technology.

Lew, A., J. E. Marsden, M. Ortiz, and M. West [2004], Variational time integrators, Intern. J. Num. Meth. in Engin. 60, 153-212. MR2073073 (2005g:74054)

Lo, M., B. G. Williams, W. E. Bollman, D. Han, Y. Hahn, J. L. Bell, E. A. Hirst, R. A. Corwin, P. E. Hong, K. C. Howell, B. Barden, and R. Wilson [2001], Genesis Mission Design, The Journal of the Astronautical Sciences 49, 169-184.

Lo, M. W. and S. D. Ross [1998], Low energy interplanetary transfers using invariant manifolds of L1, L2 and halo orbits. In AAS/AIAA Space Flight Mechanics Meeting, Monterey, California.

Lo, M. W. and S. D. Ross [2001], The Lunar L1 Gateway: Portal to the stars and beyond. In AIAA Space 2001 Conference, Albuquerque, New Mexico.

Marsden, J. E. and M. West [2001], Discrete mechanics and variational integrators, Acta Numerica 10, 357-514. MR2009697 (2004h:37130)

Meiss, J. D. [1992], Symplectic maps, variational principles, and transport. Rev. Mod. Phys. 64 (3), 795-848. MR1183196 (93h:58060)

Meyer, K. R. and R. Hall [1992], Hamiltonian Mechanics and the N-Body Problem. Texts in Applied Mathematics Science. Springer-Verlag, Berlin.

Monien, B., R. Preis, and R. Diekmann [2000], Quality matching and local improvement for multilevel graph-partitioning. Parallel Computing, 26 (12), 1609-1634. MR1786939 (2001d:68181)

Poincaré, H. [1890], Sur le problème des trois corps et les équations de la dynamique, Acta Math. 13, 1-27. 
Poincaré, H. [1892], Les Méthodes Nouvelles de la Mécanique Celeste. 3 volumes. English translation, New Methods of Celestial Mechanics, History of Modern Physics and Astronomy 13, Amer. Inst. Phys., 1993.

Porter, M.A. and P. Cvitanović [2005], Ground control to Niels Bohr: Exploring outerspace with atomic physics, Notices of the AMS 52 (October 2005), 10201025.

Rom-Kedar, V. [1999], Transport in a class of $n$-d.o.f. systems, in Hamiltonian systems with three or more degrees of freedom (S'Agaró, 1995), Vol. 533 of NATO Adv. Sci. Inst. Ser. C Math. Phys. Sci., Kluwer Acad. Publ., Dordrecht, 538-543. MR1720944 (2000k:37088)

Rom-Kedar, V. and S. Wiggins [1990], Transport in two-dimensional maps. Arch. Rat. Mech. Anal. 109, 239-298. MR1025172 (90j:58137)

Ross, S. D. [2003], Statistical theory of interior-exterior transition and collision probabilities for minor bodies in the solar system, in Libration Point Orbits and Applications, World Scientific, 637-652.

Ross, S. D. [2005], A mechanism for capture, escape, and collision in dynamical astronomy (in preparation).

Ross, S. D., W. S. Koon, M. W. Lo, and J. E. Marsden [2003], Design of a Multi-Moon Orbiter. In 13th AAS/AIAA Space Flight Mechanics Meeting, Ponce, Puerto Rico. Paper No. AAS 03-143.

Roy, A. E. [1988], Orbital Motion. Adam Hilger, Bristol, 3rd edition.

Serban, R., W. S. Koon, M. Lo, J. E. Marsden, L. R. Petzold, S. D. Ross, and R. S. Wilson [2002], Halo orbit mission correction maneuvers using optimal control, Automatica 38, 571-583. MR2131468 (Review)

Wiggins, S. [1992], Chaotic transport in dynamical systems. Interdisciplinary Appl. Math. 2. Springer, Berlin-Heidelberg-New York. MR1139113 (93c:58152)

Control and Dynamical Systems, California Institute of Technology 107-81, PasaDENA, CALIFORNIA 91125

E-mail address: marsden@cds.caltech.edu

Department of Aerospace and Mechanical Engineering, University of Southern California, RRB 217, Los Angeles, California 90089-1191

E-mail address: s.ross@usc.edu 